\title{
Molecular Mechanism of ISC Iron-Sulfur Cluster Biogenesis Revealed by High-Resolution Native Mass Spectrometry
}

\author{
Cheng-Wei Lin, \\ Department of Chemistry, Texas A\&M University, College Station, Texas 77842, United States \\ Jacob W. McCabe, \\ Department of Chemistry, Texas A\&M University, College Station, Texas 77842, United States \\ David H. Russell, \\ Department of Chemistry, Texas A\&M University, College Station, Texas 77842, United States \\ David P. Barondeau \\ Department of Chemistry, Texas A\&M University, College Station, Texas 77842, United States
}

\begin{abstract}
Iron-sulfur $(\mathrm{Fe}-\mathrm{S})$ clusters are ubiquitous protein cofactors that are required for many important biological processes including oxidative respiration, nitrogen fixation, and photosynthesis. Biosynthetic pathways assemble $\mathrm{Fe}-\mathrm{S}$ clusters with different iron-to-sulfur stoichiometries and distribute these clusters to appropriate apoproteins. In the ISC pathway, the pyridoxal $5^{\prime}$ phosphate-dependent cysteine desulfurase enzyme IscS provides sulfur to the scaffold protein IscU, which templates the Fe-S cluster assembly. Despite their functional importance, mechanistic details for cluster synthesis have remained elusive. Recent advances in native mass spectrometry (MS) have allowed proteins to be preserved in native-like structures and support applications in the investigation of protein structure, dynamics, ligand interactions, and the identification of proteinassociated intermediates. Here, we prepared samples under anaerobic conditions and then applied native MS to investigate the molecular mechanism for $\mathrm{Fe}-\mathrm{S}$ cluster synthesis. This approach was validated by the high agreement between native MS and traditional visible circular dichroism spectroscopic assays. Time-dependent native MS experiments revealed potential iron- and sulfurbased intermediates that decay as the $[2 \mathrm{Fe}-2 \mathrm{~S}]$ cluster signal developed. Additional experiments establish that (i) $\mathrm{Zn}(\mathrm{II})$ binding stabilizes IscU and protects the cysteine residues from oxidation, weakens the interactions between IscU and IscS, and inhibits Fe-S cluster biosynthesis; and (ii) $\mathrm{Fe}$ (II) ions bind to the IscU active site cysteine residues and another lower affinity binding site and promote the intermolecular sulfur transfer reaction from IscS to IscU. Overall, these results
\end{abstract}

Corresponding Author: barondeau@tamu.edu.

The authors declare no competing financial interest.

ASSOCIATED CONTENT

Supporting Information

The Supporting Information is available free of charge at https://pubs.acs.org/doi/10.1021/jacs.9b11454.

Additional tables showing the predicted and observed mass of IscU species during cluster formation; mass shifts for ${ }^{34} \mathrm{~S}-\mathrm{L}-\mathrm{cysteine}$ labeled species; figures showing the drift time distribution of apo-IscU in monomeric and dimeric forms, dimerization studies of IscU, representative native MS spectra upon combining $\mathrm{Zn}$-IscU and IscS, selected spectra for the time-dependent native MS experiment, native MS data for a separate reaction under rapid cluster assembly conditions; native MS data for mixtures of apo-IscU and Zn-IscU (PDF) 
support an iron-first model for Fe-S cluster synthesis and highlight the power of native MS in defining protein-associated intermediates and elucidating mechanistic details of enzymatic processes.

\section{Graphical Abstract}

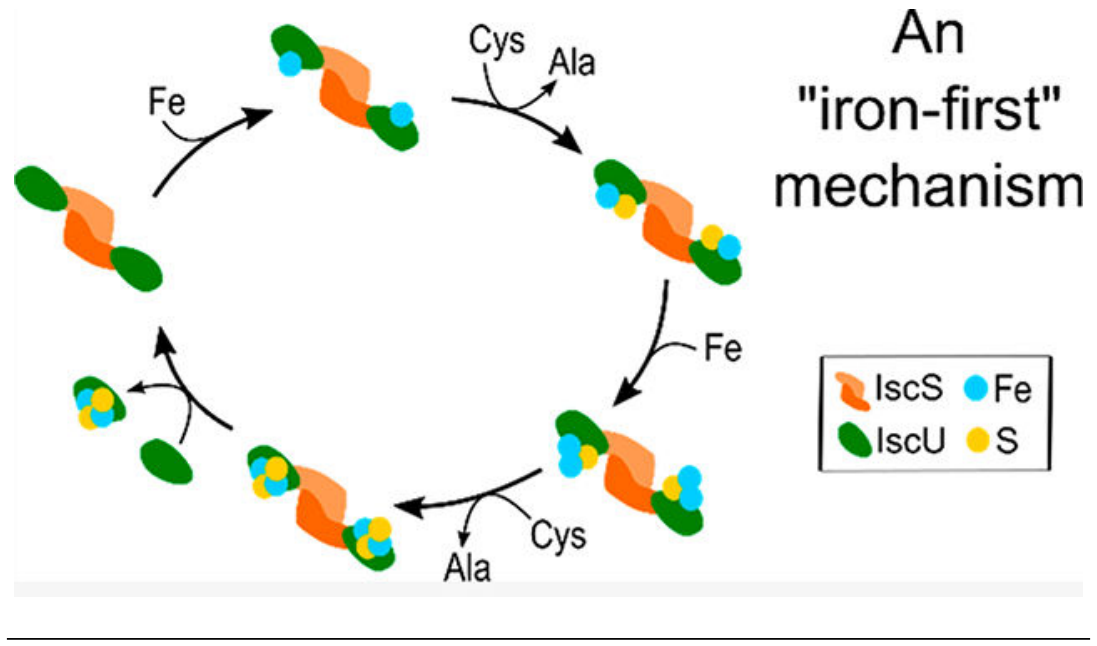

\section{INTRODUCTION}

Iron-sulfur $(\mathrm{Fe}-\mathrm{S})$ clusters are protein cofactors that are necessary for critical cellular processes such as oxidative respiration, photosynthesis, nitrogen fixation, and DNA replication/repair. Fe-S cluster cofactors exhibit a range of functional roles including electron transfer, substrate binding and activation, small molecule sensing, and controlling activity through regulation at the DNA, RNA, and protein levels. ${ }^{1,2} \mathrm{Fe}-\mathrm{S}$ clusters have a variety of iron-to-sulfur stoichiometries and are sometimes coupled to other metal cofactors. 3-5 The most common species found in proteins are the rhombic $[2 \mathrm{Fe}-2 \mathrm{~S}]$ and cubic $[4 \mathrm{Fe}-$ 4S] forms, which may be the building blocks for other Fe-S cofactors. Despite their physiological importance, few mechanistic details are known about intermediates in the formation and transformation of these $\mathrm{Fe}-\mathrm{S}$ species.

The ISC (iron-sulfur cluster) biosynthetic pathway is found in many bacteria and in the mitochondria of eukaryotes. ${ }^{6-8}$ In the bacterial pathway, the cysteine desulfurase IscS converts the substrate L-cysteine into L-alanine and provides the sulfur to the scaffold protein IscU for $\mathrm{Fe}-\mathrm{S}$ cluster assembly. ${ }^{9-11}$ The requirement of an iron donor protein and the source of iron are not clear. ${ }^{12-15}$ The IscU-IscS complex can build [2Fe-2S] and, possibly, [4Fe-4S] cluster intermediates from $\mathrm{Fe}^{2+}$, L-cysteine, and an electron source. ${ }^{16-18}$ Ferredoxin is reported to be the electron donor, ${ }^{19}$ but it is not essential and can be substituted with reagents such as glutathione (GSH) for in vitro $\mathrm{Fe}-\mathrm{S}$ cluster synthesis assays. ${ }^{20}$ To complete the catalytic cycle for the assembly complex, the Fe-S cluster intermediates are transferred intact to the recipient proteins by the assistance of chaperone and/or carrier proteins. ${ }^{21-26}$

Crystal structures of the IscU-IscS complex with and without a [2Fe-2S] cluster reveal key protein-protein interactions and provide insight into the intermolecular sulfur transfer 
reaction. ${ }^{27,28}$ IscS exists as a stable homodimer with each subunit containing a $5^{\prime}$-pyridoxal phosphate (PLP) cofactor and a mobile S-transfer loop cysteine residue. IscU subunits bind to opposite ends of the IscS dimer to form an overall $a_{2} \beta_{2}$ IscU-IscS quaternary structure. Sulfur can be transferred as a persulfide species from IscS to IscU. ${ }^{11}$ To transfer sulfur, the mobile loop of IscS oscillates a distance of $\sim 14 \AA$ between the PLP and conserved cysteine residues on IscU. ${ }^{27,28} \mathrm{IscU}$ is often referred to as a metamorphic protein and exists as an equilibrium mixture of structured and disordered states. ${ }^{29}$ This equilibrium can be shifted toward the structured state upon binding of either $\mathrm{Zn}^{2+}$ or $\mathrm{Fe}^{2+} .{ }^{29-31}$ Structural studies reveal that both $\mathrm{Zn}^{2+}$ and $[2 \mathrm{Fe}-2 \mathrm{~S}]$ clusters are ligated to $\mathrm{Isc} \mathrm{U}$ by its three cysteine residues. 28,32 Early studies also revealed that uncomplexed IscU can dimerize and undergo a cluster synthesis sequence with one $[2 \mathrm{Fe}-2 \mathrm{~S}]^{2+}$, two $[2 \mathrm{Fe}-2 \mathrm{~S}]^{2+}$, and then one $[4 \mathrm{Fe}-4 \mathrm{~S}]^{2+}$ cluster bound per dimeric protein. ${ }^{16}$ Finally, aerobically grown crystals of the IscU-IscS complex revealed an oxidized $\mathrm{Fe}_{2} \mathrm{~S}-\mathrm{S}$ species in which one sulfur both bridges the two iron atoms and also forms a disulfide bond with a second dangling sulfur atom. ${ }^{28}$ It is unclear if this species represents an intermediate in cluster formation or is simply an oxidative degradation product. Overall, the lack of characterized intermediates or even the order of sulfur and iron incorporation events have limited our mechanistic understanding of $\mathrm{Fe}-\mathrm{S}$ cluster biosynthesis.

In this study, we applied time-resolved native mass spectrometry (MS) to probe the formation of intermediate species in the synthesis of $\mathrm{Fe}-\mathrm{S}$ clusters on the scaffold protein IscU. Visible circular dichroism (CD) spectroscopy is the current state-of-the-art method of monitoring $[2 \mathrm{Fe}-2 \mathrm{~S}]$ cluster formation in biosynthetic assays. ${ }^{33-35}$ However, this technique and other spectroscopic approaches have offered few details into the formation and decay of intermediates in this Fe-S cluster assembly process. Native MS has been used for studying protein structure, dynamics, ligand interactions, and, more recently, the time-dependent tracking of protein-associated $\mathrm{Fe}-\mathrm{S}$ intermediates for the oxygen-sensing protein FNR. ${ }^{36-45}$ Native MS takes advantage of soft ionization techniques and careful instrument tuning to minimize collisional activation and preserve proteins in their folded native-like structure. $41,42,46$ Here, we show that the kinetics of [2Fe-2S]-IscU formation monitored by native MS coincides well with that from a traditional visible CD spectroscopic assay. In addition, we provide evidence for potential intermediates and a long-sought mechanism for the synthesis of $\mathrm{Fe}-\mathrm{S}$ clusters by the ISC system.

\section{RESULTS}

\section{Characterization of IscU and IscS}

High-resolution native MS was implemented to investigate the sequence of events in the assembly of $\mathrm{Fe}-\mathrm{S}$ clusters by the ISC biosynthetic pathway. The E. coli cysteine desulfurase IscS, which had typical activity in a sulfide generation assay $(8.6 \pm 0.2 \mu \mathrm{M}$ sulfide/min $\cdot \mu \mathrm{M}$ $\mathrm{IscS}$ ), and the scaffold protein IscU were purified to homogeneity. Both IscS and IscU were then exchanged into a volatile ammonium acetate buffer that is compatible with native MS experiments. IscS, which is a homodimer with an expected molecular mass of 97,032.3 Da including the His6-tags and PLP cofactors, exhibited a mass of 97,029.0 $\pm 20.3 \mathrm{Da}$. IscU had an observed molecular mass of 13,976.0 $\pm 7.0 \mathrm{Da}$ in the apo form (expected mass of 
$13,976.7 \mathrm{Da}$ ) and could form an $\mathrm{Isc}_{2} \mathrm{IscS}_{2}$ species with IscS (observed molecular mass of $124,972.6 \pm 23.6 \mathrm{Da}$; expected total mass of $124,985.7 \mathrm{Da}$ ). Both IscS (cysteine desulfurase activity $=2.0 \pm 0.1 \mu \mathrm{M}$ sulfide/min $\mu \mathrm{M}$ IscS) and IscU (in a Fe-S cluster assembly assay; see below) were functional under the native MS buffer conditions.

\section{$\mathrm{Zn}^{2+}$ and $\mathrm{Fe}^{2+}$ Binding to IscU}

Native MS analysis of as-isolated IscU revealed a predominantly monomeric form (>95\%) with a mass of 14,038.9 \pm 7.9 Da, which is consistent with one $\mathrm{Zn}^{2+}$ bound to IscU (Figure $1 \mathrm{~A}, \mathrm{~B}$; red trace). Treatment with the DTPA chelator resulted in a mass reduction of $62.9 \mathrm{Da}$ $\left(\mathrm{Zn}^{2+}-2 \mathrm{H}^{+}\right.$, expected mass reduction of $\left.63.4 \mathrm{Da}\right)$, indicating conversion to apo-IscU (Figures 1A, 1B; black trace). Different ratios (1:2, 1:1, and 2:1) of apo-IscU and Zn-IscU mixtures match well with the relative abundances in the native MS spectra (Figure S1), suggesting that the abundance of species detected by MS reflects the relative concentrations in solution. Ion mobility MS revealed that apo-IscU exhibited single compact peaks for charge states, which indicates native-like protein folds (Figure S2). Exposure to aerobic conditions slowly converted apo-IscU from a monomeric to a dimeric form (Figure S3). Titration of IscU samples exhibiting mixed oligomeric states (approximate monomer-todimer ratio of 78:22 for apo-IscU and 86:14 for $\mathrm{Zn}-\mathrm{IscU}$ ) with $\mathrm{Fe}^{2+}$ revealed that apo-IscU binds a single $\mathrm{Fe}^{2+}$ at lower concentrations and a second $\mathrm{Fe}^{2+}$ at higher ( 220 -fold molar excess) concentrations (Figure 1C, D). The relative normalized intensities of unbound and Fe-bound IscU suggests that monomeric IscU has a higher binding affinity for $\mathrm{Fe}^{2+}$ than dimeric IscU (Figure 1D). $\mathrm{Zn}-\mathrm{IscU}$ bound one $\mathrm{Zn}^{2+}$ per subunit, which was not displaced by the addition of $\mathrm{Fe}^{2+}$ (Figure 1E), suggesting that $\mathrm{Fe}^{2+}$ cannot readily displace IscU-bound $\mathrm{Zn}^{2+}$. The addition of excess iron ( $\geq 20$-fold molar excess) to $\mathrm{Zn}-\mathrm{IscU}$ generated a species containing both $\mathrm{Zn}^{2+}$ and $\mathrm{Fe}^{2+}$. Moreover, the addition of $\mathrm{Fe}^{2+}$ resulted in the development of a positive ellipticity feature at $315 \mathrm{~nm}$ in CD spectra when added to apo-IscU, but not $\mathrm{Zn}-\mathrm{IscU}$ (Figure 1F), consistent with the native MS results (Figure 1D, E). Overall, these results indicate that each IscU molecule is capable of binding up to two metal ions and that $\mathrm{Fe}^{2+}$ cannot readily displace the $\mathrm{Zn}^{2+}$ under these conditions.

\section{The Dimerization of IscU Is Promoted by Oxidation of the Active Site Cysteine Residues}

Next, we used native MS to determine the amount of dimeric IscU after incubation of either apo-IscU or $\mathrm{Zn}-\mathrm{IscU}$ under anaerobic or aerobic conditions and in the presence or absence of reducing agents (Figure S3). Aerobic conditions induced dimerization of both apo-IscU and $\mathrm{Zn}-\mathrm{IscU}$, consistent with disulfide formation involving one of the three IscU cysteines located in the $[2 \mathrm{Fe}-2 \mathrm{~S}]$ cluster assembly active site. Dimerization through intermolecular disulfide formation is supported by the ability of cysteine-ligated $\mathrm{Zn}^{2+}$ to reduce the propensity to dimerize under both anaerobic and aerobic conditions and by the ability of the reducing agent TCEP to dramatically lower the formation of dimeric IscU under both anaerobic and aerobic conditions (Figure S3). Consistent with this result, nonreducing SDSPAGE analysis demonstrated that dimeric IscU was diminished after treatment with TCEP (Figure S4A). We also observed that both DTT and GSH inhibited the dimerization of apoIsc $\mathrm{U}$ and $\mathrm{Zn}-\mathrm{IscU}$ under anaerobic conditions but promoted dimerization in an aerobic environment. The increase in dimerization under aerobic conditions may be linked to the ability of the oxidized form of these sulfur-containing reagents to facilitate disulfide bond 
formation. Thus, IscU dimers are mediated by intermolecular disulfide bonds between active site cysteine residues.

\section{The Influence of $\mathrm{Zn}^{2+}$ and $\mathrm{Fe}^{2+}$ on the Binding Affinity of IscU to IscS}

We next investigated the effect of metal binding on the formation of the $\mathrm{IscU}_{2}-\mathrm{IscS}_{2}$ complex using native MS (Figure 2). Combining apo-IscU with IscS resulted in a large fraction (94\%) of the $\mathrm{IscU}_{2}-\mathrm{IscS}_{2}$ complex and a small fraction (6\%) of an IscU-IscS species (Figure 2B). The addition of a 2-fold molar excess of $\mathrm{Fe}^{2+}$ resulted in the binding of two $\mathrm{Fe}^{2+}$ per $\mathrm{IscU}_{2}-\mathrm{IscS}_{2}$ complex and slightly decreased the fraction $(86 \%)$ of $\mathrm{IscU}_{2}-\mathrm{IscS}_{2}$ species (Figure 2A; apo-IscU + IscS $+\mathrm{Fe}^{2+}$ ). In contrast, combining $\mathrm{Zn}-\mathrm{IscU}$ with IscS resulted in a much larger fraction (47\%) of incompletely associated IscU-IscS 2 complex with a moderate fraction (11\%) of $\mathrm{IscS}_{2}$ entirely lacking IscU (Figures $2 \mathrm{~B}, \mathrm{~S} 5$ ). The $\mathrm{Zn}^{2+}$ remained bound upon the formation of the complex with IscS (Figure 2A; Zn-IscU + IscS). The addition of $\mathrm{Fe}^{2+}$ resulted in the binding of an extra $\mathrm{Fe}^{2+}$ per IscU without displacing the $\mathrm{Zn}^{2+}$ ion (Figure $2 \mathrm{~A}, \mathrm{Zn}-\mathrm{IscU}+\mathrm{IscS}+\mathrm{Fe}^{2+}$ ). Adding a mixture of apo and $\mathrm{Zn}$-bound IscU to IscS generated an intermediate amount of the IscU-IscS $S_{2}$ complex with slightly enhanced binding of $\mathrm{Zn}-\mathrm{IscU}$ to IscS (Figure 2B). Overall, our results reveal that IscU-IscS complexes are capable of uptaking two metal ions per IscU with different affinities, $\mathrm{Zn}^{2+}$ binds tighter than $\mathrm{Fe}^{2+}$ to IscU, $\mathrm{Zn}^{2+}$ decreases the binding affinity of IscU to IscS, and $\mathrm{Fe}^{2+}$ has a minor effect on the binding affinity of IscU to IscS.

\section{$\mathrm{Zn}^{2+}$ Binding Inhibits Cluster Formation on IscU}

To investigate the effect of $\mathrm{Zn}^{2+}$ binding to Isc $\mathrm{U}$ on [2Fe-2S] cluster formation, cluster assembly under the conditions of low IscS, L-cysteine, and GSH was monitored by CD spectroscopy. A characteristic CD signal for [2Fe-2S]-IscU was observed with apo-IscU (Figure 3A), but not Zn-IscU (Figure 3B). Consistently, the apo-IscU sample developed a [2Fe-2S]-IscU signal when monitored by native MS, but the $\mathrm{Zn}-\mathrm{IscU}$ sample showed neither $\mathrm{Zn}^{2+}$ displacement nor simultaneous binding of both $\mathrm{Zn}^{2+}$ and a [2Fe-2S] cluster (data not shown; see additional experiments below).

Since $\mathrm{Zn}^{2+}$ could function as a physiological inhibitor, we investigated how $\mathrm{Zn}^{2+}$ might be displaced from IscU using native MS (Figure 3C). The $\mathrm{Zn}^{2+}$ ion was not removed by a 30 min incubation of $\mathrm{Zn}$-IscU with L-cysteine, DTT, GSH, IscS, $\mathrm{Fe}^{2+}$, or IscS plus $\mathrm{Fe}^{2+}$ (Figure 3C). In contrast, a 5 min incubation of $\mathrm{Zn}$-IscU with IscS and L-cysteine resulted in the loss of the $\mathrm{Zn}^{2+}$ ion and formation of a persulfide species on IscU. Note that the concentration of IscS (equimolar with IscU) and the resulting cysteine desulfurase activity was much higher in the $\mathrm{Zn}$-removal experiment than in the $\mathrm{CD}$ [2Fe-2S] cluster assembly assay (Figure 3B). Thus, $\mathrm{Zn}^{2+}$ is an inhibitor of Fe-S cluster assembly on $\mathrm{IscU}$ and is not readily displaced by $\mathrm{Fe}^{2+}$ but can be removed from Isc $\mathrm{U}$ with either a $\mathrm{Zn}$ chelator or by cysteine turnover using an equimolar IscS concentration.

\section{$\mathrm{Fe}^{2+}$ Promotes Sulfur Transfer to IscU}

Here, we evaluated the influence of $\mathrm{Fe}^{2+}$ on the transfer of sulfur from IscS to IscU using time-dependent acid-quench MS experiments. Previously published studies establish that the addition of acid quenches sulfur transfer reactions and locks the persulfide label in place for 
subsequent analysis. ${ }^{47,48}$ Apo-IscU, IscS, L-cysteine, and various concentrations of $\mathrm{Fe}^{2+}$ were reacted, quenched with acid at different reaction times, and analyzed by MS. In a $3 \mathrm{~min}$ reaction, very little persulfide formation was detected on $\mathrm{IscU}$ in the absence of $\mathrm{Fe}^{2+}$ (Figure $4 \mathrm{~A}$ ), whereas multiple persulfide species were observed upon the addition of $\mathrm{Fe}^{2+}$ (Figure $4 \mathrm{~B})$. After a $1 \mathrm{~h}$ reaction, samples with and without $\mathrm{Fe}^{2+}$ had about the same amount of persulfide label ( 1.3) per IscU (Figure 4C). However, the initial rate of sulfur accumulation on IscU increased by approximately 6-fold in the presence of $400 \mu \mathrm{M} \mathrm{Fe}^{2+}$ ions. These experiments indicate that the addition of $\mathrm{Fe}^{2+}$ promotes sulfur transfer from IscS to IscU.

\section{Mechanism of Cluster Formation}

Next, we used time-dependent native MS experiments to track the formation and decay of $\mathrm{Fe}$ $-\mathrm{S}$ cluster assembly intermediates. The $\mathrm{Fe}-\mathrm{S}$ biosynthetic reaction was initiated in an anaerobic glovebox by combining apo-IscU, IscS, L-cysteine, $\mathrm{Fe}^{2+}$, and GSH.

Representative raw MS spectra collected during cluster formation revealed peaks associated with monomeric (1500-2500 m/ $\mathrm{z}$ window) and dimeric (2500-4000 $\mathrm{m} / \mathrm{z}$ window) $\mathrm{Isc}$ (Figure 5A). The raw MS spectra were deconvoluted to generate normalized intensities and assign intermediates (Figures 5B, S6). Well-resolved peaks were observed in all of the collected native MS spectra with low amounts of sodium adducts, which enabled unambiguous assignment. The predicted masses and observed mass shifts matched well for the assigned species (Table S1). Further evidence for the assignment of sulfur-based species was provided by repeating the $\mathrm{Fe}-\mathrm{S}$ assembly reactions with ${ }^{34} \mathrm{~S}$-labeled L-cysteine and comparing the difference in the mass shifts for the normal and isotope-labeled reactions (Table S2). A nearly identical time course for the development and decay of the [2Fe-2S] cluster was observed for a reaction monitored by both native MS and CD spectroscopy (Figure 5C).

In the first 2 min of the reaction, the amounts of monomeric $\mathrm{Fe}-\mathrm{IscU}, \mathrm{Fe}-\mathrm{S}-\mathrm{IscU}$, and $2 \mathrm{Fe}-$ IscU species increased dramatically and decreased in concentration at later times as the amount of monomeric [2Fe-2S]-IscU increased (Figure 5D, left), consistent with being cluster assembly intermediates. Similarly, the concentrations of S-IscU and $2 \mathrm{~S}-\mathrm{IscU}$ species also increased rapidly to a concentration that only gradually decreased over the remaining $165 \mathrm{~min}$ of the reaction (Figure 5D, middle). The lack of rapid decay of these one and two sulfur-bound species either suggests that they are off-pathway products or that they are intermediates that reach a steady state during the reaction. Low levels of IscU containing three and four sulfur atoms were also observed at longer reaction times.

The monomeric [2Fe-2S]-IscU reached a maximum after $75 \mathrm{~min}$ and then decreased, and similar kinetics were also observed for the low levels of monomeric $2 \mathrm{Fe}-\mathrm{IscU}$ and $2 \mathrm{Fe} 1 \mathrm{~S}-$ IscU species (Figure 5D, left). As the [2Fe-2S]-IscU concentration decreased at longer reaction times, we observed the development of monomeric $2 \mathrm{Fe} 3 \mathrm{~S}-\mathrm{Isc} \mathrm{U}$ and dimeric $4 \mathrm{Fe} X \mathrm{~S}-\mathrm{IscU}(X=6-8)$ species (Figure 5C, D; Tables S1, S7). Interestingly, a separate reaction using excess substrates and the nonphysiological reductant DTT generated traditional [4Fe-4S] clusters and not the $4 \mathrm{Fe} X \mathrm{~S}-\mathrm{Isc} \mathrm{U}$ species (Figure S8). In general, dimers of IscU both bound to and lacking Fe-S clusters accumulated at later time points (Figures 5D, right, S7). Overall, these native MS experiments suggest a pathway for the 
development of [2Fe-2S] clusters on IscU with additional conversion chemistry generating clusters with larger stoichiometries at longer reaction times.

\section{DISCUSSION}

Elaborate biosynthetic pathways are responsible for assembling $\mathrm{Fe}-\mathrm{S}$ clusters and delivering the appropriate cluster form to clientele proteins. Although there are decades of excellent studies of the self-assembly of Fe-S clusters and the associated enzymatic process, ${ }^{49,50}$ key mechanistic questions remain. Here, we implemented high-resolution time-dependent native MS experiments to define intermediates and provide insight into the assembly of $\mathrm{Fe}-\mathrm{S}$ clusters for the bacterial ISC biosynthetic system.

Native MS experiments separate unreacted substrates from iron- and sulfur-based intermediates, which can be identified without relying on model compounds or samples that are chiral, labeled, or paramagnetic. To ensure that the protein ions were not collisionally activated, which might result in the loss of labile intermediates, and that the proteins remained in native-like conformations, ${ }^{46,51,52}$ ion mobility measurements were performed using a homemade source and a drift tube coupled to an EMR instrument. ${ }^{53,54}$ These experiments revealed protein ions that were in a compact native-like conformation and charge state distributions that were suitable for further data processing (Figure S2).

Additional evidence that native MS is appropriate for deciphering solution $\mathrm{Fe}-\mathrm{S}$ cluster chemistry is provided by the high agreement for the formation and decay of [2Fe-2S]-IscU monitored by native MS and CD spectroscopy (Figure 5C). The reaction conditions for these experiments were designed to be consistent with the time resolution and measurement requirements of the MS experiment. A separate MS reaction was performed that verified that the $[2 \mathrm{Fe}-2 \mathrm{~S}]$ clusters survive the nano-ESI process (Figure S8). We also provide evidence supporting the correlation of species abundances in solution (mole fraction) with that detected by native MS (normalized intensity). The monomer to dimer ratio of an oxidized IscU sample investigated by native MS (59 $\pm 5 \%$ dimer) or nonreducing SDS-PAGE followed by densitometry (65 $\pm 6 \%$ dimer) are in agreement (Figure S4). Also, different ratios (1:2, 1:1, and 2:1) of apo-IscU and $\mathrm{Zn}$-IscU mixtures match well with the relative abundances in the native MS spectrum (Figure S1). Moreover, the highly purified proteins, high mass resolution, and ${ }^{34} \mathrm{~S}$ isotope labeling experiments enable unambiguous assignment of the intermediate species associated with IscU (Figure 5; Tables S1, S2). Collectively, the data presented in this study highlight the power of native MS in defining protein-associated intermediates and elucidating details of enzymatic reactions.

Native MS experiments revealed that the as-isolated Isc $\mathrm{U}$ has one $\mathrm{Zn}^{2+}$ bound per protein (Figure 1B) and is able to form a $\mathrm{Zn}$-bound $\mathrm{Isc}_{2}-\mathrm{IscS}_{2}$ complex (Figure 2B). $\mathrm{Zn}^{2+}$ also inhibited [2Fe-2S] cluster formation on IscU (Figure 3), consistent with previous studies that $\mathrm{Zn}^{2+}$ stabilizes IscU by binding to the same cysteine residues that are used to ligate $[2 \mathrm{Fe}-2 \mathrm{~S}]$ cluster intermediates. ${ }^{28,32,55-58} \mathrm{Zn}^{2+}$ binding stabilizes uncomplexed Isc $\mathrm{U}^{58}$ and, in our experiments, decreases the propensity of the IscU subunits to dimerize (Figure S3). Reductants also decrease IscU dimerization (Figures S3, S4A), indicating that cysteine residues are being oxidized to form an intermolecular disulfide bond. The ability of $\mathrm{Zn}^{2+}$ to protect cysteine residues from oxidation has been reported for other proteins. ${ }^{59}$ Although 
uncomplexed IscU copurifies with $\mathrm{Zn}^{2+}$, the cellular metal-binding status of IscU under in vivo conditions is unclear as $E$. coli is reported to have extremely low free $\mathrm{Zn}^{2+}$ concentrations. ${ }^{60}$ Moreover, native MS results indicate apo-IscU binds tighter than the $\mathrm{Zn}$ bound form to IscS (Figure 2B). Regardless, $\mathrm{Zn}^{2+}$ can be removed from $\mathrm{Zn}-\mathrm{IscU}$ with the chelating agent DTPA (Figure 1B) ${ }^{58,61}$ or by reactions that include both L-cysteine and a stoichiometric amount of IscS (Figure 3C), which might remove the $\mathrm{Zn}^{2+}$ either by generating a persulfide species on IscU that decreases the $\mathrm{Zn}^{2+}$ binding affinity or by precipitating the $\mathrm{Zn}^{2+}$ with generated sulfide. Overall, the $\mathrm{Zn}^{2+}$ stabilizes and protects IscU from oxidation and remains bound until the IscS-dependent turnover of L-cysteine promotes the loss of $\mathrm{Zn}^{2+}$ and initiates the Fe-S cluster assembly reaction.

Native MS experiments revealed that apo-IscU can bind two $\mathrm{Fe}^{2+}$ ions with different affinities (Figure 1D), consistent with recent Mössbauer spectroscopic studies that show two $\mathrm{Fe}^{2+}$ binding sites for human ISCU with distinct chemical environments. ${ }^{61}$ Moreover, $\mathrm{Fe}-$ IscU exhibited a positive ellipticity peak in the $\mathrm{CD}$ spectrum at $315 \mathrm{~nm}$ that is characteristic of a $\mathrm{S} \rightarrow$ Fe charge transfer band (Figure 1F). Recent studies on human Fe-IscU describe a similar spectroscopic feature and provide mutagenesis experiments that support cysteine ligation for the $\mathrm{Fe}^{2+}$ ion. ${ }^{61} \mathrm{In}$ addition, we provide evidence that $\mathrm{Fe}^{2+}$ is unable to displace the $\mathrm{Zn}^{2+}$ (Figures 1C, 3C) but can bind simultaneously with $\mathrm{Zn}^{2+}$ to IscU (Figure 1E), likely to the lower affinity site. Notably, the three cysteine residues on IscU are located in the $[2 \mathrm{Fe}-2 \mathrm{~S}]$ cluster assembly active site. These combined results indicate that IscU has two metal-binding sites: a higher affinity site that binds $\mathrm{Zn}^{2+}$ and $\mathrm{Fe}^{2+}$ using the active site cysteines and a lower affinity site that binds $\mathrm{Fe}^{2+}$. It is unclear if both iron-binding sites are functionally relevant, but it is important to note that two $\mathrm{Fe}^{2+}$ ions are required to synthesize a $[2 \mathrm{Fe}-2 \mathrm{~S}]$ cluster.

We also found that the addition of $\mathrm{Fe}^{2+}$ stimulates the reactivity of the cysteine residues on IscU. Acid-quench denaturing MS experiments revealed that $\mathrm{Fe}^{2+}$ accelerates sulfur accumulation on apo-IscU (Figure 4), consistent with a recent study for the human assembly system. ${ }^{61}$ Previous experiments using the bacterial IscU-IscS complex indicate that $\mathrm{Fe}^{2+}$ has no effect on the PLP chemistry or cysteine desulfurase activity, ${ }^{62}$ which suggests that $\mathrm{Fe}^{2+}$ instead promotes the intermolecular sulfur transfer reaction. The stimulatory role of $\mathrm{Fe}^{2+}$ in sulfur transfer might be explained by (i) decreasing the $\mathrm{p} K_{\mathrm{a}}$ of an IscU sulfur-accepting cysteine ligand to increase its nucleophilicity; (ii) functioning as a Lewis acid to increase the electrophilicity of the sulfane sulfur of the IscS persulfide; and (iii) inducing a local conformational change to properly position the sulfur donor and acceptor residues. Also, native MS experiments revealed that reactions that include $\mathrm{Fe}^{2+}$ incorporated oxygen to generate low levels of sulfenic acid (Table S1). Notably, the formation of small amounts of sulfinic acid, which would have the same mass as a persulfide species, might explain the lower than expected mass shifts for reactions with ${ }^{34} \mathrm{~S}$-L-cysteine (Table S2). These results are consistent with the ability of redox-active metals such as iron to enhance the reactivity of cysteine ligands. ${ }^{59,63}$ 


\section{Model for Fe-S Cluster Assembly}

The Fe-S cluster assembly reaction likely occurs in the context of the IscU-IscS complex. Substrates for the synthesis of $\mathrm{Fe}-\mathrm{S}$ clusters on IscU are delivered through IscS or proteins that bind with the $\mathrm{Isc}_{2}-\mathrm{IscS}_{2}$ complex. ${ }^{10,12,13,19,27}$ One of the challenges in using native MS to investigate intermediates in Fe-S cluster assembly is the $a_{2} \beta_{2}$ nature of the IscU-IscS complex; i.e., two Fe-S species bound to separate IscU subunits would have the same total mass as the $\mathrm{IscU}_{2}-\mathrm{IscS}_{2}$ complex with one bound [2Fe-2S] cluster. We, therefore, designed experiments to probe uncomplexed $\mathrm{IscU}$ in the reaction with the underlying assumption that the resulting iron and sulfur bound species are a readout of intermediates on the IscU-IscS complex. Fe-S assembly reactions were initiated by mixing apo-IscU, $\mathrm{Fe}^{2+}$, L-cysteine, IscS, and $\mathrm{GSH}$, and the reactions were monitored by measuring changes in the mass of uncomplexed $\mathrm{IscU}$ as a function of time.

The MS data suggest an "iron-first" mechanism for [2Fe-2S] cluster synthesis (Figure 6). Our results reveal a $[2 \mathrm{Fe}-2 \mathrm{~S}]$ cluster bound to monomeric IscU developed, maximized after $75 \mathrm{~min}$, and then decreased in intensity (Figure 5D). This trace coincided well with the positive ellipticity at $330 \mathrm{~nm}$ associated with [2Fe-2S]-IscU, which maximized at an estimated $18 \mu \mathrm{M},{ }^{20}$ in a parallel assay monitored by CD spectroscopy (Figure 5C).

Monomeric Isc U with shifts in mass consistent with $\mathrm{Fe}$ and $\mathrm{Fe}-\mathrm{S}$ species were observed in the earliest time points that then decayed as the intensity of the $[2 \mathrm{Fe}-2 \mathrm{~S}]$ cluster peak increased. This behavior is consistent with the $\mathrm{Fe}-\mathrm{IscU}$ and $\mathrm{Fe}-\mathrm{S}-\mathrm{IscU}$ species being intermediates in the $[2 \mathrm{Fe}-2 \mathrm{~S}]$ cluster assembly reaction (Figure 6). The $\mathrm{Fe}-\mathrm{IscU}$ peak decayed before the $\mathrm{Fe}-\mathrm{S}-\mathrm{IscU}$ species, suggesting a major route to a [2Fe-2S] cluster is through an iron-first mechanism. This hypothesis is also supported by the acceleration of the intermolecular sulfur transfer reaction from IscS to IscU in the presence of $\mathrm{Fe}^{2+}$ (Figure 4). As the $\mathrm{Fe}-\mathrm{S}-\mathrm{IscU}$ species decays, signals for both $2 \mathrm{Fe}-1 \mathrm{~S}-\mathrm{Isc} \mathrm{U}$ and $[2 \mathrm{Fe}-2 \mathrm{~S}]-\mathrm{IscU}$ develop. Therefore, we propose that an iron atom binds to the Fe-S-IscU species prior to the second intermolecular sulfur transfer reaction. The two iron atoms are likely oxidized from $\mathrm{Fe}^{2+}$ to $\mathrm{Fe}^{3+}$ to provide the two electrons required to break the S-S bond of the persulfide, ${ }^{64}$ generate a bridging sulfide intermediate, and freeing the cysteine residue on IscU to accept another persulfide from IscS.

We propose an oxidized $2 \mathrm{Fe}-2 \mathrm{~S}$ species is then generated after a second intermolecular sulfur transfer reaction (Figure 6). This species may be reminiscent of the $\mathrm{Fe}_{2} \mathrm{~S}-\mathrm{S}$ oxidized cluster observed in a crystal structure of the IscU-IscS complex ${ }^{28}$ and needs to be reduced by two electrons to complete the $[2 \mathrm{Fe}-2 \mathrm{~S}]^{2+}$ assembly process. These electrons appear to be provided through the oxidation of ferredoxin in vivo and glutathione in our in vitro assay. We include GSH in our reactions because it is a physiological reagent that promotes in vitro $[2 \mathrm{Fe}-2 \mathrm{~S}]$ cluster synthesis and transfer reactions to cluster carrier and target proteins. ${ }^{20}$ Although ferredoxin clearly has a role in $[2 \mathrm{Fe}-2 \mathrm{~S}]$ and/or $[4 \mathrm{Fe}-4 \mathrm{~S}]$ cluster biogenesis in vivo, ${ }^{65}$ the ability of bacterial $\mathrm{Fdx}$ to compete with $\mathrm{IscU}$ for binding to $\mathrm{IscS}$ and also generate a persulfide radical anion on $\mathrm{IscS}^{19}$ appear counterproductive to a role in [2Fe-2S] cluster synthesis and require further study.

There may be additional viable routes with lower efficiency that also form a [2Fe-2S] cluster. A diiron species is observed at early time points that immediately decays and then 
develops again coincidental with the [2Fe-2S]-IscU signal. It is possible that this diiron species is an intermediate that incorporates sulfur to generate the same $2 \mathrm{Fe}-1 \mathrm{~S}$ intermediate described above (Figure 6). A "sulfur-first" mechanism is also possible, as a peak associated with a single persulfide bound to IscU develops at the earliest time points and then gradually decays over the entire time course of the experiment. The incorporation of iron would generate the Fe-S-IscU intermediate that could then proceed through the rest of the assembly process. We view this as a minor route toward [2Fe-2S] cluster formation as the initial rate of persulfide formation on Isc $\mathrm{U}$ is stimulated by about 6 -fold in the presence of $\mathrm{Fe}^{2+}$ (Figure 4).

We also observe IscU species that lack iron but have incorporated multiple sulfur atoms during in vitro cluster formation reactions. The number of persulfide sulfur atoms associated with monomeric (up to 4) and dimeric (up to 3) IscU increases during the reaction (Figure $5 \mathrm{C}$, right panel), consistent with previous observations. ${ }^{11,66,67} \mathrm{We}$ do not include $2 \mathrm{~S}-\mathrm{IscU}$ as a potential intermediate since it would most likely need to go through a $\mathrm{Fe}-2 \mathrm{~S}-\mathrm{IscU}$ species, which was not observed in the time-dependent experiment, to generate [2Fe-2S]-IscU. Glutathione associated adducts on IscU with up to 2 persulfide sulfur atoms were also detected (Table $\mathrm{S} 1)$. In addition, larger polypersulfide IscU species $(\mathrm{S}>2)$ were observed but have not been demonstrated to be viable intermediates in Fe-S cluster assembly and may be dead-end products.

As the reaction proceeds, monomeric IscU is replaced by dimeric IscU (Figures 5D, S7), likely in a cysteine-mediated oxidation process (Figures S3, S4A), and larger-order Fe-S clusters are generated. At longer reaction times, the amount of [2Fe-2S]-IscU decreased and the formation of $2 \mathrm{Fe}-3 \mathrm{~S}$ species on monomeric IscU and higher-order 4Fe- $X \mathrm{~S}(X=6-9)$ clusters on dimeric IscU increased. The higher-order clusters are likely generated by coupling chemistry between monomeric IscU molecules, similar to the previously described DTT-mediated reductive coupling of two [2Fe-2S]-IscU subunits to generate [4Fe-4S]$\mathrm{Isc}_{2} \cdot{ }^{16,68} \mathrm{We}$ were also able to detect $[4 \mathrm{Fe}-4 \mathrm{~S}]-\mathrm{Isc}_{2}$ using native $\mathrm{MS}$ in a separate DTTmediated reaction (Figure S8). Interestingly, the formation of the higher-order clusters explains the decrease of [2Fe-2S]-IscU CD features at longer reaction times under these conditions (Figure 5C). It is currently unclear if the reductive coupling of [2Fe-2S]-IscU species is a physiological route toward generating $[4 \mathrm{Fe}-4 \mathrm{~S}]$ clusters.

In summary, we use native MS experiments to investigate the $\mathrm{Fe}-\mathrm{S}$ cluster biogenesis process of the bacterial ISC system. We provide details for $\mathrm{Zn}^{2+}$ and $\mathrm{Fe}^{2+}$ binding to IscU, the influence of metal binding on the IscU-IscS complex stoichiometry, the $\mathrm{Zn}$-dependent inhibition of cluster synthesis, the IscS-mediated removal of $\mathrm{Zn}^{2+}$, the oxidative dimerization of $\mathrm{IscU}$, and the ability of $\mathrm{Fe}^{2+}$ to enhance the intermolecular sulfur transfer reaction from IscS to IscU. In addition, we contribute time-dependent native MS experiments aimed at identifying intermediates in the biosynthesis of Fe-S clusters and propose a mechanism consistent with our findings. Overall, this work highlights how native MS can be applied as a powerful tool in mechanistic enzymology by providing details of protein complex formation, protein-ligand interactions, and the identity and time-dependent tracking of reaction intermediates. 


\section{MATERIALS AND METHODS}

\section{Preparation of Proteins}

Escherichia coli proteins IscS and IscU were expressed and purified as previously described.

62 A pET-16a plasmid containing the cysteine synthase (CysK1) gene from Mycobacterium tuberculosis (gift of T. Begley, Texas A\&M University) was transformed into E. coli BL21 (DE3) cells. The expressed CysK1 contained an N-terminal 6-His affinity tag and a Factor Xa protease site. CysK1 was purified using the same Ni-NTA and size exclusion chromatography columns and procedures used for the IscS purification. An extinction coefficient of $6.6 \mathrm{mM}^{-1} \mathrm{~cm}^{-1}$ at $388 \mathrm{~nm}$ (in $0.1 \mathrm{M} \mathrm{NaOH}$ ) was used to estimate the concentration of the PLP cofactor, ${ }^{69}$ which represents the concentration of active IscS. An extinction coefficient of $11,460 \mathrm{M}^{-1} \mathrm{~cm}^{-1}$ at $280 \mathrm{~nm}$ was used to estimate the concentrations of both IscU and CysK1. IscS, IscU, and CysK1 were estimated to be $>95 \%$ pure based on SDS-PAGE analysis. Aliquots of IscS ( $605 \mu \mathrm{M} ; 50 \mathrm{mM}$ HEPES, $250 \mathrm{mM} \mathrm{NaCl}, \mathrm{pH}=7.5$ ), IscU (301 $\mu \mathrm{M} ; 50 \mathrm{mM}$ HEPES, $150 \mathrm{mM} \mathrm{NaCl}, \mathrm{pH}=8.0)$, and CysK1 $(1078 \mu \mathrm{M} ; 50 \mathrm{mM}$ HEPES, $150 \mathrm{mM} \mathrm{NaCl}, \mathrm{pH}=8.0$ ) stock solutions were flash-frozen and stored at $-80{ }^{\circ} \mathrm{C}$. All samples were prepared in an anaerobic Mbraun glovebox $\left(<1 \mathrm{ppm}\right.$ of $\mathrm{O}_{2}$ monitored by a Teledyne model 310 analyzer) unless otherwise indicated.

\section{Sample Preparation for MS Experiments}

Protein samples were desalted by two passes through Micro Biospin columns with Bio-gel P-6 (Bio-Rad) equilibrated with target MS-compatible ammonium acetate buffers. Under standard conditions, Isc $\mathrm{U}$ copurified with $\mathrm{Zn}^{2+}(\mathrm{Zn}-\mathrm{Isc} \mathrm{U})$ and was prepared for native MS by desalting the IscU stock solution after purification. Apo-IscU was prepared by treating the IscU stock solution with $10 \mathrm{mM}$ DTPA for $30 \mathrm{~min}$ and then desalting the sample. To minimize potential oxidation during MS analysis, all samples were prepared and incubated in $0.2 \mathrm{~mL}$ PCR tubes in the glovebox. The reaction mixtures were then sealed in the PCR tubes, removed from the glovebox, and infused within approximately 1 min into the mass spectrometer.

\section{Monitoring $\mathrm{Fe}-\mathrm{IscU}$ and [2Fe-2S]-IscU by CD Spectroscopy}

For CD measurements, the samples were prepared in a $1 \mathrm{~cm}$ path length anaerobic cuvette and sealed with a rubber septum and parafilm in the glovebox. Spectra were recorded at room temperature on a CD spectrometer (Applied Photophysics Chirascan). To evaluate $\mathrm{Fe}^{2+}$ binding to IscU, a $50 \mu \mathrm{M}$ apo-IscU or $\mathrm{Zn}-\mathrm{IscU}$ sample was mixed with $500 \mu \mathrm{M}$ ferrous acetate in buffer $\mathrm{A}(200 \mathrm{mM}$ ammonium acetate, $\mathrm{pH}=6.8)$ and incubated at room temperature for $30 \mathrm{~min}$ prior to measuring the $\mathrm{CD}$ signal. To monitor [2Fe-2S]-IscU cluster formation, either $100 \mu \mathrm{M} \mathrm{Zn-IscU}$ or apo-IscU was mixed with $3 \mu \mathrm{M} \mathrm{IscS,} 200 \mu \mathrm{M} \mathrm{L}$ cysteine, $100 \mu \mathrm{M} \mathrm{GSH}$, and $500 \mu \mathrm{M}$ ferrous acetate in buffer A.

\section{Native MS Experiments to Monitor Metal Binding to IscU}

A titration of Isc $\mathrm{U}$ with $\mathrm{Fe}^{2+}$ was monitored by native MS experiments. A concentration of $10 \mu \mathrm{M}$ apo-IscU or $\mathrm{Zn}-\mathrm{Isc} \mathrm{U}$ was mixed with different concentrations of ferrous acetate (50, 
$100,200,400$, or $800 \mu \mathrm{M}$ ) in buffer B (900 mM ammonium acetate, $20 \mathrm{mM}$ ammonium bicarbonate, $\mathrm{pH}=6.8$ ), incubated for $30 \mathrm{~min}$, and then analyzed by native MS.

\section{Native MS Experiments to Monitor IscU Dimerization}

A concentration of $10 \mu \mathrm{M}$ apo-IscU or Zn-IscU was mixed with either $1 \mathrm{mM}$ D,L-DTT, 200 $\mu \mathrm{M}$ GSH, or $1 \mathrm{mM}$ TCEP in buffer B. A sample was immediately removed (labeled initial) for MS analysis. Additional samples were incubated for $36 \mathrm{~h}$ at $25^{\circ} \mathrm{C}$ in the glovebox or under aerobic conditions prior to MS analysis.

\section{Native MS Experiments to Monitor IscU-IscS Complex Formation}

To assess the formation and stoichiometry of IscU-IscS complexes, $50 \mu \mathrm{M} \mathrm{IscS} \mathrm{was} \mathrm{mixed}$ with $50 \mu \mathrm{M}$ apo-IscU, $50 \mu \mathrm{M}$ Zn-IscU, $25 \mu \mathrm{M}$ apo-IscU and $25 \mu \mathrm{M} \mathrm{Zn}-\mathrm{IscU}, 50 \mu \mathrm{M}$ apoIsc $\mathrm{U}$ and $100 \mu \mathrm{M} \mathrm{Fe}^{2+}$, or $50 \mu \mathrm{M} \mathrm{Zn-IscU}$ and $100 \mu \mathrm{M} \mathrm{Fe}^{2+}$ in buffer $\mathrm{B}$. The mixtures were incubated for $30 \mathrm{~min}$ before the native MS measurement.

\section{Native MS Experiments to Monitor $\mathrm{Zn}^{2+}$ Displacement}

To monitor $\mathrm{Zn}^{2+}$ removal from IscU, $10 \mu \mathrm{M} \mathrm{Zn}-\mathrm{IscU}$ was mixed with $1 \mathrm{mM}$ L-cysteine, 1 mM D,L-DTT, $1 \mathrm{mM} \mathrm{GSH}$, or $800 \mu \mathrm{M}$ ferrous acetate in buffer A, incubated for $30 \mathrm{~min}$, and analyzed by native MS. In addition, $40 \mu \mathrm{M} Z \mathrm{Zn}-\mathrm{IscU}$ was mixed with $40 \mu \mathrm{M}$ IscS, $40 \mu \mathrm{M}$ IscS, and $200 \mu \mathrm{M} \mathrm{Fe}^{2+}$, or $40 \mu \mathrm{M}$ IscS and $200 \mu \mathrm{M} \mathrm{L}$-cysteine in buffer A, incubated for 5 $\mathrm{min}$, and then diluted 4 fold before analysis by native MS experiments.

\section{Time-Dependent Acid-Quench Denaturing MS Experiments}

To determine whether $\mathrm{Fe}^{2+}$ influences sulfur transfer from IscS to IscU, mixtures of $75 \mu \mathrm{M}$ apo-IscU, $15 \mu \mathrm{M}$ IscS, and different concentrations $(0,100$, or $400 \mu \mathrm{M})$ of ferrous acetate were incubated in $200 \mathrm{mM}$ ammonium acetate $(\mathrm{pH}=7.5)$ for $30 \mathrm{~min}$ at $22{ }^{\circ} \mathrm{C}$. The reaction was then initiated by adding $300 \mu \mathrm{M} \mathrm{L}$-cysteine. At each time point, the assay mixture was quenched by 10 -fold dilution into $1 \%$ formic acid. The $0.2 \mathrm{~mL}$ PCR tubes containing the quenched samples were removed from the glovebox and infused into the mass spectrometer. Linear regression fits were used to determine the initial rates for the sulfur transfer reactions.

\section{Time-Dependent Native MS Monitoring of Fe-S Cluster Assembly}

A mixture $(220 \mu \mathrm{L})$ of $3 \mu \mathrm{M}$ IscS and $100 \mu \mathrm{M}$ IscU in buffer A was first incubated in the glovebox for $30 \mathrm{~min}$ at $22{ }^{\circ} \mathrm{C}$. The reaction was then initiated by adding $200 \mu \mathrm{M} \mathrm{L}$-cysteine, $100 \mu \mathrm{M} \mathrm{GSH}$, and $250 \mu \mathrm{M}$ ferrous acetate. Fe-S cluster formation was monitored by native MS or by CD spectroscopy as a function of time. For time-dependent native MS experiments, the assay mixture was diluted 10-fold by buffer B and placed into $0.2 \mathrm{~mL}$ PCR tubes, removed from the glovebox, and immediately infused into an EMR Orbitrap instrument using a commercial nano-ESI source. The MS spectrum was collected for $30 \mathrm{~s}$. The entire process from the dilution of the assay mixture to spraying the sample into the MS instrument was completed within approximately $1 \mathrm{~min}$. In addition, the assay sample was transferred to an anaerobic cuvette in the glovebox, sealed with a cap, and brought out of the glovebox for CD measurements. 


\section{Isotope Labeling to Identify Sulfur-Containing Intermediates}

An isotope labeling experiment was performed to confirm the identity of the sulfurcontaining intermediates. ${ }^{34} \mathrm{~S}$-L-cysteine was synthesized by reacting $O$-acetyl-L-serine and ${ }^{34} \mathrm{~S}^{2-}$ with $M$. tuberculosis CysK1 using a slight modification of a previous procedure. ${ }^{70}$ $\mathrm{Na}_{2}{ }^{34} \mathrm{~S}$ was prepared by using sodium metal to reduce ${ }^{34} \mathrm{~S}$ (CIL Inc.) in dry THF with a catalytic amount $(7.5 \mathrm{~mol} \%)$ of naphthalene. ${ }^{71}{ }^{34} \mathrm{~S}$-L-cysteine was synthesized by mixing $70 \mu \mathrm{M}$ CysK1 with $20 \mathrm{mM} O$-acetyl-L-serine hydrochloride (SigmaAldrich) and $\sim 10 \mathrm{mM}$ $\mathrm{Na}_{2}{ }^{34} \mathrm{~S}$ at $37{ }^{\circ} \mathrm{C}$ overnight in the glovebox. A $3 \mathrm{kDa}$ mass spin concentrator was used to separate ${ }^{34} \mathrm{~S}$-L-cysteine from CysK. The ${ }^{34} \mathrm{~S}$-L-cysteine concentration in the flow-through was determined by an acid-ninhydrin assay. ${ }^{72}$ The ${ }^{34}$ S-L-cysteine solution, which contained some unreacted $O$-acetylserine, was directly used for $\mathrm{Fe}-\mathrm{S}$ cluster formation assays. For the isotope labeling experiment, mixtures of $100 \mu \mathrm{M}$ apo-IscU, $3 \mu \mathrm{M} \mathrm{IscS,}, 200 \mu \mathrm{M}{ }^{34} \mathrm{~S}-\mathrm{L}$ cysteine, $100 \mu \mathrm{M} \mathrm{GSH}$, and $250 \mu \mathrm{M}$ ferrous acetate in buffer A were reacted for $40 \mathrm{~min}$ in a glovebox. At the targeted reaction time, the isotope labeling Fe-S cluster formation assay mixture was quenched and cleaned by a Micro Biospin column with Bio-gel P-6 previously equilibrated with buffer A. Samples were then removed from the glovebox for native MS measurements. For a peak containing $\mathrm{N}$ sulfur atoms, the expected mass shift would be $\left[34\left(\right.\right.$ mass of $\left.{ }^{34} \mathrm{~S}\right)-32.1$ (average mass of natural-abundance sulfur) $] \times \mathrm{N}=1.9 \mathrm{~N}$.

\section{Native MS Description and Settings}

Gold-coated borosilicate glass capillaries were prepared in house for use in the nano-ESI experiments. The sample solution was backfilled into a gold-coated glass capillary tip and sprayed into the mass spectrometer. Native MS experiments were performed by an Exactive Plus with extended mass range (EMR) Orbitrap MS (Thermo Fisher Scientific, San Jose, $\mathrm{CA})$. Instrument parameters were tuned to minimize collisional activation while retaining reasonable signal-to-noise. The ion mobility experiments were performed on a homemade instrument, Fourier transform-ion mobility-EMR (FT-IMS-EMR), with previously described experimental conditions. ${ }^{53,54}$ Specifically, the measurements were conducted with a frequency sweep of $5-5005 \mathrm{~Hz}$ in $8 \mathrm{~min}$ at approximately 1.725 Torr $\mathrm{He}$ in the drift tube.

Reasonable simultaneous transmission for monomeric and dimeric IscU was obtained by tuning the DC voltages on the ion lenses and transport multipoles and using a lower resolution setting $(R=8750)$. The parameters used for the native MS analysis of IscU include: $\mathrm{m} / \mathrm{z}$ range $1000-5000$, spray voltage $1.5 \mathrm{kV}$, capillary temperature $50-100{ }^{\circ} \mathrm{C}, \mathrm{S}$ Lens RF level 200, source DC offset $25 \mathrm{~V}$, injection flatapole DC $16 \mathrm{~V}$, inter flatapole lens DC $12 \mathrm{~V}$, bent flatapole DC $8 \mathrm{~V}$, transfer multipole DC offset $4 \mathrm{~V}$, C-trap entrance lens tune offset $0 \mathrm{~V}$, trapping gas pressure setting 7, in-source dissociation voltage $0 \mathrm{eV}, \mathrm{HCD}$ collision energy $10 \mathrm{eV}$, FT resolution 8750, positive ion mode, and ion maximum injection time $200 \mathrm{~ms}$.

The acid-quench denaturing MS parameters used for the analysis of the persulfide IscU species include: $\mathrm{m} / \mathrm{z}$ range $500-5000$, spray voltage $1.3 \mathrm{kV}$, capillary temperature $250{ }^{\circ} \mathrm{C}, \mathrm{S}$ Lens RF level 200, source DC offset $25 \mathrm{~V}$, injection flatapole DC $16 \mathrm{~V}$, inter flatapole lens DC $12 \mathrm{~V}$, bent flatapole DC $8 \mathrm{~V}$, transfer multipole DC offset $2 \mathrm{~V}$, C-trap entrance lens tune offset $0 \mathrm{~V}$, trapping gas pressure setting 2, in-source dissociation voltage $0 \mathrm{eV}, \mathrm{HCD}$ 
collision energy $0 \mathrm{eV}$, FT resolution 70,000, positive ion mode, and ion maximum injection time $50 \mathrm{~ms}$.

The native MS parameters used for the analysis of the IscU-IscS complex include: $\mathrm{m} / \mathrm{z}$ range 4000-9000, spray voltage $1.5 \mathrm{kV}$, capillary temperature $125^{\circ} \mathrm{C}$, S-Lens RF level 200, source DC offset $25 \mathrm{~V}$, injection flatapole DC $16 \mathrm{~V}$, inter flatapole lens DC $12 \mathrm{~V}$, bent flatapole DC $8 \mathrm{~V}$, transfer multipole DC offset $9 \mathrm{~V}$, C-trap entrance lens tune offset $0 \mathrm{~V}$, trapping gas pressure setting 7, in-source dissociation voltage $160 \mathrm{eV}, \mathrm{HCD}$ collision energy $10 \mathrm{eV}$, FT resolution 35,000, positive ion mode, and ion maximum injection time $200 \mathrm{~ms}$.

\section{MS Data Processing}

The initial MS data were collected using the Thermo Exactive software as RAW format. The raw data were converted into text files using a Python script making use of Multiplierz. The intensities of the protein and protein-adduct species were deconvoluted and converted into normalized intensities by processing the text files with the software program UniDec. ${ }^{73}$ The intact mass for the different species was obtained from processing the native MS spectrum, and the errors were calculated from fwhm of the highest intensity peak with UniDec. Lower charge states (higher $\mathrm{m} / \mathrm{z}$ ) of IscU showed native-like conformations in ion mobility experiments. Ranges that were chosen for further data processing include $m / z$ of $2300-2400$ (native monomeric IscU), $\mathrm{m} / z$ of $3080-4000$ (native dimeric IscU), $\mathrm{m} / z$ 1500-4000 (both native monomeric and dimeric IscU), $\mathrm{m} / z$ of $800-3500$ (denaturing IscU), and $\mathrm{m} / z$ of 4000 9000 (native IscU-IscS complexes). For the time-dependent native MS experiments, the intensity of individual IscU species was further converted to the normalized intensity of individual monomeric or dimeric IscU species relative to the total amount of IscU.

\section{Supplementary Material}

Refer to Web version on PubMed Central for supplementary material.

\section{ACKNOWLEDGMENTS}

We are indebted to Prof. Tadhg Begley for use of the CD instrument and for the CysK plasmid. We are grateful to Prof. Daniel Singleton and Kai-Yuan Kuan for their assistance in preparing ${ }^{34}$ S-L-cysteine. We also wish to thank Dr. James Vranish for pilot studies and Prof. Arthur Laganowsky, Dr. Yang Liu, Dr. Michael Poltash, Dr. Seth Cory, Dr. Shachin Patra, and Dr. Christopher Putnam for helpful discussions and technical assistance.

Funding

This work was supported in part by NIH Grants R01GM096100 (D.P.B.), R01GM121751 (D.H.R.), P41GM128577 (D.H.R.), NSF Grant CHE 1508269 (D.P.B.), and the Robert A. Welch Grant A-1647 (D.P.B.).

\section{ABBREVIATIONS}

$\begin{array}{ll}\text { MS } & \text { mass spectrometry } \\ \text { ISC } & \text { iron-sulfur cluster } \\ \text { PLP } & 5^{\prime} \text {-pyridoxal phosphate } \\ \text { CD } & \text { circular dichroism }\end{array}$




$\begin{array}{ll}\text { CysK1 } & \text { cysteine synthase } \\ \text { EMR } & \text { Exactive Plus with extended mass range } \\ \text { fwhm } & \text { full width at half-maximum } \\ \text { DC } & \text { direct current } \\ \text { RF } & \text { radio frequency } \\ \text { HCD } & \text { Higher-energy C-trap dissociation } \\ \text { FT } & \text { Fourier transform }\end{array}$

\section{REFERENCES}

(1). Johnson DC; Dean DR; Smith AD; Johnson MK Structure, function, and formation of biological iron-sulfur clusters. Annu. Rev. Biochem. 2005, 74, 247-81. [PubMed: 15952888]

(2). Py B; Barras F Building Fe-S proteins: bacterial strategies. Nat. Rev. Microbiol. 2010, 8 (6), 43646. [PubMed: 20467446]

(3). Drennan CL; Peters JW Surprising cofactors in metalloenzymes. Curr. Opin. Struct. Biol. 2003, 13 (2), 220-6. [PubMed: 12727516]

(4). Beinert H Iron-sulfur proteins: ancient structures, still full of surprises. JBIC, J. Biol. Inorg. Chem. 2000, 5 (1), 2-15. [PubMed: 10766431]

(5). Beinert H; Holm RH; Munck E Iron-sulfur clusters: nature's modular, multipurpose structures. Science 1997, 277 (5326), 653-9. [PubMed: 9235882]

(6). Frazzon J; Dean DR Formation of iron-sulfur clusters in bacteria: an emerging field in bioinorganic chemistry. Curr. Opin. Chem. Biol. 2003, 7 (2), 166-73. [PubMed: 12714048]

(7). Zheng L; Cash VL; Flint DH; Dean DR Assembly of iron-sulfur clusters. Identification of an iscSUA-hscBA-fdx gene cluster from Azotobacter vinelandii. J. Biol. Chem. 1998, 273 (21), 13264-72. [PubMed: 9582371]

(8). Muhlenhoff U; Lill R Biogenesis of iron-sulfur proteins in eukaryotes: a novel task of mitochondria that is inherited from bacteria. Biochim. Biophys. Acta, Bioenerg. 2000, 1459 (23), 370-82.

(9). Zheng L; White RH; Cash VL; Dean DR Mechanism for the desulfurization of L-cysteine catalyzed by the nifS gene product. Biochemistry 1994, 33 (15), 4714-20. [PubMed: 8161529]

(10). Cupp-Vickery JR; Urbina H; Vickery LE Crystal structure of IscS, a cysteine desulfurase from Escherichia coli. J. Mol. Biol. 2003, 330 (5), 1049-59. [PubMed: 12860127]

(11). Smith AD; Agar JN; Johnson KA; Frazzon J; Amster IJ; Dean DR; Johnson MK Sulfur transfer from IscS to IscU: the first step in iron-sulfur cluster biosynthesis. J. Am. Chem. Soc. 2001, 123 (44), 11103-4. [PubMed: 11686732]

(12). Kim JH; Bothe JR; Frederick RO; Holder JC; Markley JL Role of IscX in iron-sulfur cluster biogenesis in Escherichia coli. J. Am. Chem. Soc. 2014, 136 (22), 7933-42. [PubMed: 24810328]

(13). Layer G; Ollagnier-de Choudens S; Sanakis Y; Fontecave M Iron-sulfur cluster biosynthesis: characterization of Escherichia coli CYaY as an iron donor for the assembly of [2Fe-2S] clusters in the scaffold IscU. J. Biol. Chem. 2006, 281 (24), 16256-63. [PubMed: 16603772]

(14). Yoon T; Cowan JA Iron-sulfur cluster biosynthesis. Characterization of frataxin as an iron donor for assembly of [2Fe-2S] clusters in ISU-type proteins. J. Am. Chem. Soc. 2003, 125 (20), 607884. [PubMed: 12785837]

(15). Wofford JD; Bolaji N; Dziuba N; Outten FW; Lindahl PA Evidence that a respiratory shield in Escherichia coli protects a low-molecular-mass Fe(II) pool from O2-dependent oxidation. J. Biol. Chem. 2019, 294 (1), 50-62. [PubMed: 30337367] 
(16). Agar JN; Krebs C; Frazzon J; Huynh BH; Dean DR; Johnson MK IscU as a scaffold for ironsulfur cluster biosynthesis: sequential assembly of [2Fe-2S] and [4Fe-4S] clusters in IscU. Biochemistry 2000, 39 (27), 7856-62. [PubMed: 10891064]

(17). Agar JN; Zheng LM; Cash VL; Dean DR; Johnson MK Role of the IscU protein in iron-sulfur cluster biosynthesis: IscS-mediated assembly of a [Fe2S2] cluster in IscU. J. Am. Chem. Soc. 2000, 122 (9), 2136-2137.

(18). Iannuzzi C; Adinolfi S; Howes BD; Garcia-Serres R; Clemancey M; Latour JM; Smulevich G; Pastore A The role of CyaY in iron sulfur cluster assembly on the E. coli IscU scaffold protein. PLoS One 2011, 6 (7), e21992. [PubMed: 21799759]

(19). Kim JH; Frederick RO; Reinen NM; Troupis AT; Markley JL [2Fe-2S]-ferredoxin binds directly to cysteine desulfurase and supplies an electron for iron-sulfur cluster assembly but is displaced by the scaffold protein or bacterial frataxin. J. Am. Chem. Soc. 2013, 135 (22), 8117-20. [PubMed: 23682711]

(20). Vranish JN; Das D; Barondeau DP Real-Time Kinetic Probes Support Monothiol Glutaredoxins As Intermediate Carriers in Fe-S Cluster Biosynthetic Pathways. ACS Chem. Biol. 2016, 11 (11), 3114-3121. [PubMed: 27653419]

(21). Hoff KG; Silberg JJ; Vickery LE Interaction of the iron-sulfur cluster assembly protein IscU with the Hsc66/Hsc20 molecular chaperone system of Escherichia coli. Proc. Natl. Acad. Sci. U. S. A. 2000, 97 (14), 7790-5. [PubMed: 10869428]

(22). Hoff KG; Cupp-Vickery JR; Vickery LE Contributions of the LPPVK motif of the iron-sulfur template protein IscU to interactions with the Hsc66-Hsc20 chaperone system. J. Biol. Chem. 2003, 278 (39), 37582-9. [PubMed: 12871959]

(23). Bonomi F; Iametti S; Morleo A; Ta D; Vickery LE Facilitated transfer of IscU-[2Fe2S] clusters by chaperone-mediated ligand exchange. Biochemistry 2011, 50 (44), 9641-50. [PubMed: 21977977]

(24). Chandramouli K; Johnson MK HscA and HscB stimulate [2Fe-2S] cluster transfer from IscU to apoferredoxin in an ATP-dependent reaction. Biochemistry 2006, 45 (37), 11087-95. [PubMed: 16964969]

(25). Bonomi F; Iametti S; Morleo A; Ta D; Vickery LE Studies on the mechanism of catalysis of ironsulfur cluster transfer from IscU[2Fe2S] by HscA/HscB chaperones. Biochemistry 2008, 47 (48), 12795-801. [PubMed: 18986169]

(26). Shakamuri P; Zhang B; Johnson MK Monothiol glutaredoxins function in storing and transporting [Fe2S2] clusters assembled on IscU scaffold proteins. J. Am. Chem. Soc. 2012, 134 (37), 15213-6. [PubMed: 22963613]

(27). Shi R; Proteau A; Villarroya M; Moukadiri I; Zhang L; Trempe JF; Matte A; Armengod ME; Cygler M Structural basis for Fe-S cluster assembly and tRNA thiolation mediated by IscS protein-protein interactions. PLoS Biol. 2010, 8 (4), No. e1000354. [PubMed: 20404999]

(28). Marinoni EN; de Oliveira JS; Nicolet Y; Raulfs EC; Amara P; Dean DR; Fontecilla-Camps JC (IscS-IscU) 2 complex structures provide insights into Fe2S2 biogenesis and transfer. Angew. Chem., Int. Ed. 2012, 51 (22), 5439-42.

(29). Kim JH; Tonelli M; Kim T; Markley JL Three-dimensional structure and determinants of stability of the iron-sulfur cluster scaffold protein IscU from Escherichia coli. Biochemistry 2012, 51 (28), 5557-63. [PubMed: 22734684]

(30). Kim JH; Tonelli M; Markley JL Disordered form of the scaffold protein IscU is the substrate for iron-sulfur cluster assembly on cysteine desulfurase. Proc. Natl. Acad. Sci. U. S. A. 2012, 109 (2), 454-9. [PubMed: 22203963]

(31). Markley JL; Kim JH; Dai Z; Bothe JR; Cai K; Frederick RO; Tonelli M Metamorphic protein IscU alternates conformations in the course of its role as the scaffold protein for iron-sulfur cluster biosynthesis and delivery. FEBS Lett. 2013, 587 (8), 1172-9. [PubMed: 23333622]

(32). Ramelot TA; Cort JR; Goldsmith-Fischman S; Kornhaber GJ; Xiao R; Shastry R; Acton TB; Honig B; Montelione GT; Kennedy MA Solution NMR structure of the iron-sulfur cluster assembly protein $\mathrm{U}$ (IscU) with zinc bound at the active site. J. Mol. Biol. 2004, 344 (2), 567-83. [PubMed: 15522305] 
(33). Fidai I; Wachnowsky C; Cowan JA Glutathione-complexed [2Fe-2S] clusters function in Fe-S cluster storage and trafficking. JBIC, J. Biol. Inorg. Chem. 2016, 21 (7), 887-901. [PubMed: 27590019]

(34). Bonomi F; Iametti S; Ta D; Vickery LE Multiple turnover transfer of [2Fe2S] clusters by the iron-sulfur cluster assembly scaffold proteins IscU and IscA. J. Biol. Chem. 2005, 280 (33), 29513-8. [PubMed: 15964837]

(35). Gao H; Subramanian S; Couturier J; Naik SG; Kim SK; Leustek T; Knaff DB; Wu HC; Vignols F; Huynh BH; Rouhier N; Johnson MK Arabidopsis thaliana Nfu2 accommodates [2Fe-2S] or [4Fe-4S] clusters and is competent for in vitro maturation of chloroplast [2Fe-2S] and [4Fe-4S] cluster-containing proteins. Biochemistry 2013, 52 (38), 6633-45. [PubMed: 24032747]

(36). Dyachenko A; Gruber R; Shimon L; Horovitz A; Sharon M Allosteric mechanisms can be distinguished using structural mass spectrometry. Proc. Natl. Acad. Sci. U. S. A. 2013, 110 (18), 7235-9. [PubMed: 23589876]

(37). Ganem B; Li YT; Henion JD Detection of noncovalent receptor-ligand complexes by mass spectrometry. J. Am. Chem. Soc. 1991, 113 (16), 6294-6296.

(38). Cubrilovic D; Haap W; Barylyuk K; Ruf A; Badertscher M; Gubler M; Tetaz T; Joseph C; Benz $\mathrm{J}$; Zenobi R Determination of protein-ligand binding constants of a cooperatively regulated tetrameric enzyme using electrospray mass spectrometry. ACS Chem. Biol. 2014, 9 (1), 218-26. [PubMed: 24128068]

(39). Gulbakan B; Barylyuk K; Zenobi R Determination of thermodynamic and kinetic properties of biomolecules by mass spectrometry. Curr. Opin. Biotechnol. 2015, 31, 65-72. [PubMed: 25173612]

(40). Katta V; Chait BT Observation of the Heme Globin Complex in Native Myoglobin by Electrospray-Ionization Mass-Spectrometry. J. Am. Chem. Soc. 1991, 113 (22), 8534-8535.

(41). Loo JA Studying noncovalent protein complexes by electrospray ionization mass spectrometry. Mass Spectrom. Rev. 1997, 16 (1), 1-23. [PubMed: 9414489]

(42). Marcoux J; Robinson CV Twenty years of gas phase structural biology. Structure 2013, 21 (9), 1541-50. [PubMed: 24010713]

(43). Liu Y; Cong X; Liu W; Laganowsky A Characterization of Membrane Protein-Lipid Interactions by Mass Spectrometry Ion Mobility Mass Spectrometry. J. Am. Soc. Mass Spectrom. 2017, 28 (4), 579-586. [PubMed: 27924494]

(44). Crack JC; Thomson AJ; Le Brun NE Mass spectrometric identification of intermediates in the O2-driven [4Fe-4S] to [2Fe-2S] cluster conversion in FNR. Proc. Natl. Acad. Sci. U. S. A. 2017, 114 (16), E3215-E3223. [PubMed: 28373574]

(45). Cong X; Liu Y; Liu W; Liang X; Russell DH; Laganowsky A Determining Membrane ProteinLipid Binding Thermodynamics Using Native Mass Spectrometry. J. Am. Chem. Soc. 2016, 138 (13), 4346-9. [PubMed: 27015007]

(46). Pacholarz KJ; Garlish RA; Taylor RJ; Barran PE Mass spectrometry based tools to investigate protein-ligand interactions for drug discovery. Chem. Soc. Rev. 2012, 41 (11), 4335-55. [PubMed: 22532017]

(47). Bailey TS; Zakharov LN; Pluth MD Understanding hydrogen sulfide storage: probing conditions for sulfide release from hydrodisulfides. J. Am. Chem. Soc. 2014, 136 (30), 10573-6. [PubMed: 25010540]

(48). Patra S; Barondeau DP Mechanism of activation of the human cysteine desulfurase complex by frataxin. Proc. Natl. Acad. Sci. U. S. A. 2019, 116 (39), 19421-19430. [PubMed: 31511419]

(49). Holm RH; Lo W Structural Conversions of Synthetic and Protein-Bound Iron-Sulfur Clusters. Chem. Rev. 2016, 116 (22), 13685-13713. [PubMed: 27933770]

(50). Braymer JJ; Lill R Iron-sulfur cluster biogenesis and trafficking in mitochondria. J. Biol. Chem. 2017, 292 (31), 12754-12763. [PubMed: 28615445]

(51). Chen SH; Russell DH How Closely Related Are Conformations of Protein Ions Sampled by IMMS to Native Solution Structures? J. Am. Soc. Mass Spectrom. 2015, 26 (9), 1433-43. [PubMed: 26115967] 
(52). Konijnenberg A; Butterer A; Sobott F Native ion mobility-mass spectrometry and related methods in structural biology. Biochim. Biophys. Acta, Proteins Proteomics 2013, 1834 (6), 1239-56.

(53). Poltash ML; McCabe JW; Shirzadeh M; Laganowsky A; Clowers BH; Russell DH Fourier Transform-Ion Mobility-Orbitrap Mass Spectrometer: A Next-Generation Instrument for Native Mass Spectrometry. Anal. Chem. 2018, 90 (17), 10472-10478. [PubMed: 30091588]

(54). Poltash ML; McCabe JW; Shirzadeh M; Laganowsky A; Russell DH Native IM-Orbitrap MS: Resolving what was hidden. TrAC, Trends Anal. Chem. 2019, 115533.

(55). Shimomura Y; Wada K; Fukuyama K; Takahashi Y The asymmetric trimeric architecture of [2Fe-2S] IscU: implications for its scaffolding during iron-sulfur cluster biosynthesis. J. Mol. Biol. 2008, 383 (1), 133-43. [PubMed: 18723024]

(56). Boniecki MT; Freibert SA; Muhlenhoff U; Lill R; Cygler M Structure and functional dynamics of the mitochondrial Fe/S cluster synthesis complex. Nat. Commun. 2017, 8 (1), 1287. [PubMed: 29097656]

(57). Fox NG; Yu X; Feng X; Bailey HJ; Martelli A; Nabhan JF; Strain-Damerell C; Bulawa C; Yue WW; Han S Structure of the human frataxin-bound iron-sulfur cluster assembly complex provides insight into its activation mechanism. Nat. Commun. 2019, 10 (1), 2210. [PubMed: 31101807]

(58). Fox NG; Martelli A; Nabhan JF; Janz J; Borkowska O; Bulawa C; Yue WW Zinc(II) binding on human wild-type ISCU and Met140 variants modulates NFS1 desulfurase activity. Biochimie 2018, 152, 211-218. [PubMed: 30031876]

(59). Wilcox DE; Schenk AD; Feldman BM; Xu Y Oxidation of zinc-binding cysteine residues in transcription factor proteins. Antioxid. Redox Signaling 2001, 3 (4), 549-64.

(60). Outten CE; O'Halloran TV Femtomolar sensitivity of metalloregulatory proteins controlling zinc homeostasis. Science 2001, 292 (5526), 2488-92. [PubMed: 11397910]

(61). Gervason S; Larkem D; Mansour AB; Botzanowski T; Muller CS; Pecqueur L; Le Pavec G; Delaunay-Moisan A; Brun O; Agramunt J; Grandas A; Fontecave M; Schunemann V; Cianferani S; Sizun C; Toledano MB; D’Autreaux B Physiologically relevant reconstitution of iron-sulfur cluster biosynthesis uncovers persulfide-processing functions of ferredoxin-2 and frataxin. Nat. Commun. 2019, 10 (1), 3566. [PubMed: 31395877]

(62). Bridwell-Rabb J; Iannuzzi C; Pastore A; Barondeau DP Effector role reversal during evolution: the case of frataxin in Fe-S cluster biosynthesis. Biochemistry 2012, 51 (12), 2506-14. [PubMed: 22352884]

(63). Poltash ML; Shirzadeh M; McCabe JW; Moghadamchargari Z; Laganowsky A; Russell DH New insights into the metal-induced oxidative degradation pathways of transthyretin. Chem. Commun. (Cambridge, U. K.) 2019, 55 (28), 4091-4094.

(64). Krebs C; Agar JN; Smith AD; Frazzon J; Dean DR; Huynh BH; Johnson MK IscA, an alternate scaffold for Fe-S cluster biosynthesis. Biochemistry 2001, 40 (46), 14069-80. [PubMed: 11705400]

(65). Lange H; Kaut A; Kispal G; Lill R A mitochondrial ferredoxin is essential for biogenesis of cellular iron-sulfur proteins. Proc. Natl. Acad. Sci. U. S. A. 2000, 97 (3), 1050-5. [PubMed: 10655482]

(66). Urbina HD; Silberg JJ; Hoff KG; Vickery LE Transfer of sulfur from IscS to IscU during Fe/S cluster assembly. J. Biol. Chem. 2001, 276 (48), 44521-6. [PubMed: 11577100]

(67). Smith AD; Frazzon J; Dean DR; Johnson MK Role of conserved cysteines in mediating sulfur transfer from IscS to IscU. FEBS Lett. 2005, 579 (23), 5236-40. [PubMed: 16165131]

(68). Chandramouli K; Unciuleac MC; Naik S; Dean DR; Huynh BH; Johnson MK Formation and properties of [4Fe-4S] clusters on the IscU scaffold protein. Biochemistry 2007, 46 (23), 6804 11. [PubMed: 17506525]

(69). Peterson EA; Sober HA Preparation of Crystalline Phosphorylated Derivatives of Vitamin-B6. J. Am. Chem. Soc. 1954, 76 (1), 169-175.

(70). Crack JC; Stewart MYY; Le Brun NE Generation of 34S-substituted protein-bound [4Fe-4S] clusters using 34S-L-cysteine. Biol. Methods Protoc. 2019, 4 (1), bpy015. [PubMed: 32395620] 
(71). So J-H; Boudjouk P; Hong HH; Weber WP Hexamethyldisilathiane In Inorganic Synthesis; Grimes RN, Ed.; John Wiley \& Sons: Hoboken, NJ, 1992; Vol. 29, pp 30-32.

(72). Gaitonde MK A spectrophotometric method for the direct determination of cysteine in the presence of other naturally occurring amino acids. Biochem. J. 1967, 104 (2), 627-33. [PubMed: 6048802]

(73). Marty MT; Baldwin AJ; Marklund EG; Hochberg GK; Benesch JL; Robinson CV Bayesian deconvolution of mass and ion mobility spectra: from binary interactions to polydisperse ensembles. Anal. Chem. 2015, 87 (8), 4370-6. [PubMed: 25799115] 
A
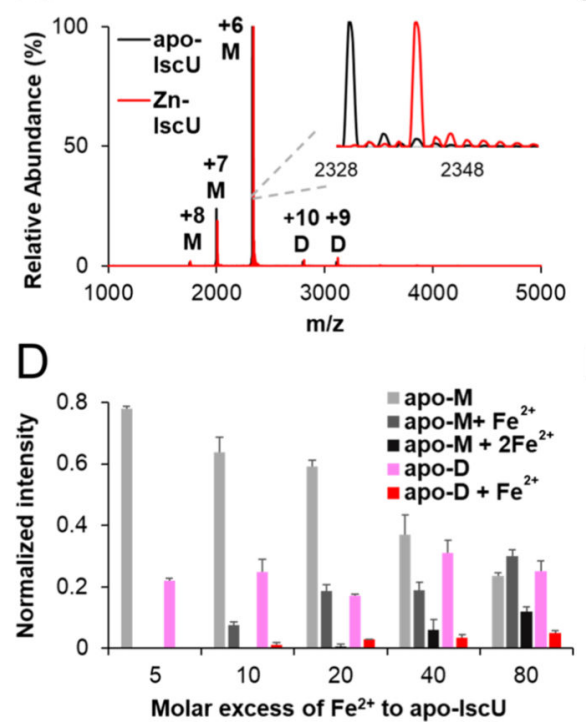

B

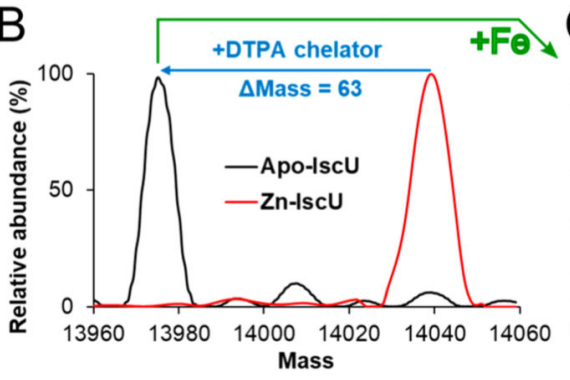

$\mathrm{E}$

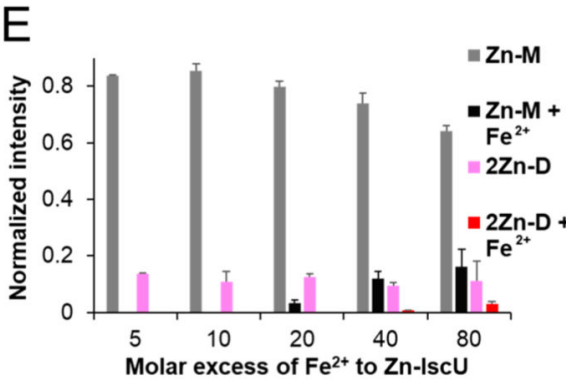

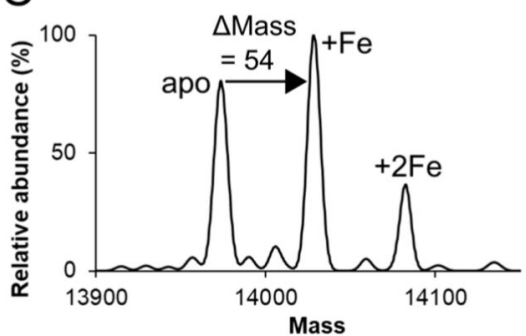

$\mathrm{F}$

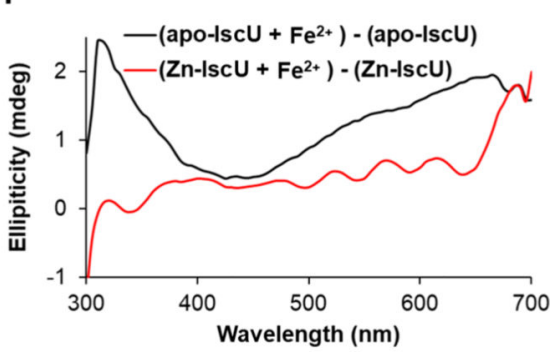

Figure 1.

Binding of $\mathrm{Zn}^{2+}$ and $\mathrm{Fe}^{2+}$ ions to IscU. (A) Raw native MS spectrum of apo-IscU (black) overlaid with as-isolated (Zn-bound) IscU (red). The $+6,+7$, and +8 charge states for monomeric IscU and the +9 and +10 charge states for dimeric IscU are shown (M: monomeric IscU, D: dimeric IscU). The inset shows the raw MS spectrum for the +6 charge state of monomeric IscU. (B) Deconvoluted zero charge MS spectrum for the +6 charge state of monomeric IscU. (C) Deconvoluted zero charge MS spectrum of $10 \mu \mathrm{M}$ apo-IscU mixed with $800 \mu \mathrm{M} \mathrm{Fe}^{2+}$. Titration of $\mathrm{Fe}^{2+}$ to $10 \mu \mathrm{M}$ apo-IscU (D) or Zn-IscU (E) monitored by native MS revealed up to two metal binding sites per IscU subunit. Both monomeric (apo-M or $\mathrm{Zn}-\mathrm{M}$ ) and dimeric (apo-D or 2Zn-D) forms of IscU were identified. The error bars are replicate errors $(n=3)$. (F) A CD spectroscopic feature at $315 \mathrm{~nm}$ develops upon the addition of $500 \mu \mathrm{M} \mathrm{Fe}^{2+}$ to apo-IscU (50 $\mu \mathrm{M}$; black) but not to Zn-IscU (50 $\mu \mathrm{M}$; red). 

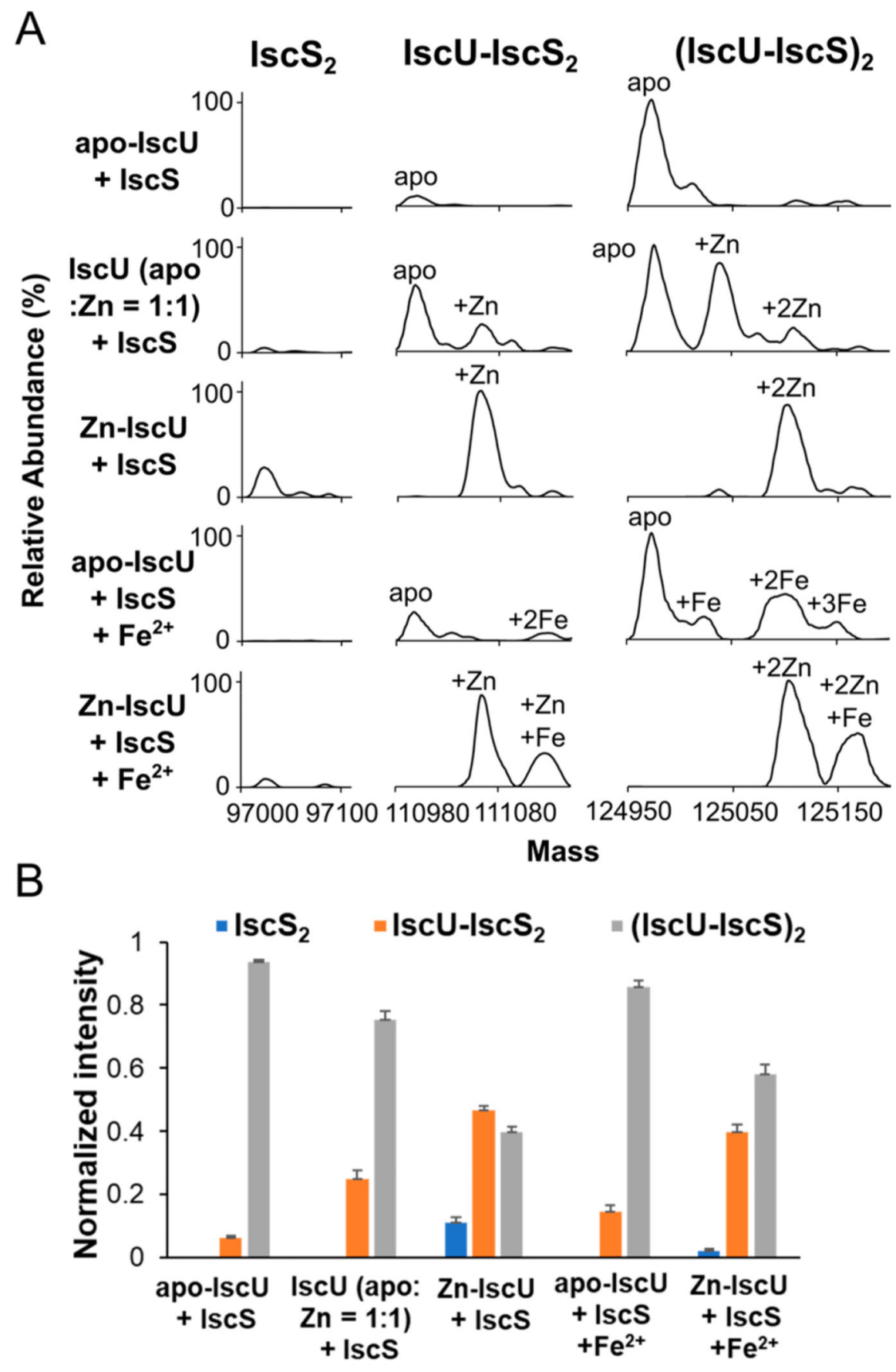

Figure 2.

Formation of apo and metal bound IscU complexes with IscS. Apo, Zn-bound, and a 1:1 mixture of the two forms of IscU (final concentration of $50 \mu \mathrm{M}$ ) were separately combined with IscS $(50 \mu \mathrm{M})$ in either the presence or absence of $\mathrm{Fe}^{2+}(100 \mu \mathrm{M})$. (A) Deconvoluted zero charge MS spectra for mass ranges that correspond to $\mathrm{IscS}_{2}(\sim 97,000 \mathrm{Da}), \mathrm{IscU}-\mathrm{IscS}_{2}$ $(\sim 111,000 \mathrm{Da})$, and $\mathrm{IscU}_{2}-\mathrm{IscS}_{2}(\sim 125,000 \mathrm{Da})$ complexes. (B) Comparison of the different protein complex stoichiometries between IscU and IscS for the five experiments. Protein 
complexes with apo and metal bound forms of IscU are combined to determine the normalized intensity. The error bars are replicate errors $(n=3)$. 

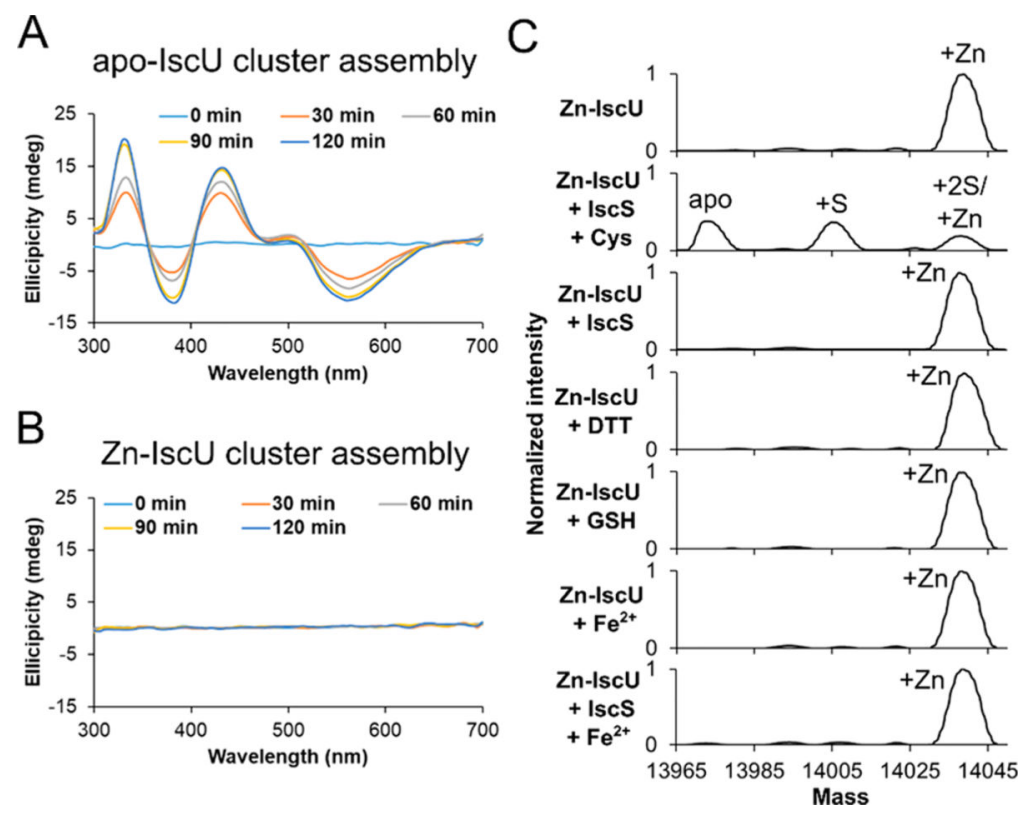

Figure 3.

$\mathrm{Zn}^{2+}$ inhibits $\mathrm{Fe}-\mathrm{S}$ assembly activity and is removed by IscS-mediated cysteine turnover. Parallel cluster formation reactions using (A) $\mathrm{Zn}-\mathrm{IscU}$ or (B) apo-IscU were monitored by CD spectroscopy. Experimental conditions: $100 \mu \mathrm{M}$ IscU, $3 \mu \mathrm{M}$ IscS, $200 \mu \mathrm{M}$ L-cysteine, $100 \mu \mathrm{M}$ GSH, and $500 \mu \mathrm{M}$ ferrous acetate. (C) Deconvoluted zero charge spectra for removal of $\mathrm{Zn}^{2+}$ from $\mathrm{Zn}$-IscU $(10 \mu \mathrm{M})$ monitored by native MS after incubations with Lcysteine $(1 \mathrm{mM})$, D,L-DTT $(1 \mathrm{mM})$, and GSH $(200 \mu \mathrm{M})$. Similar experiments were performed after incubation of $\mathrm{Zn}-\mathrm{IscU}(40 \mu \mathrm{M})$ with IscS $(40 \mu \mathrm{M})$, ferrous acetate (800 $\mu \mathrm{M})$, IscS $(40 \mu \mathrm{M})$ plus $\mathrm{Fe}^{2+}(200 \mu \mathrm{M})$, and IscS $(40 \mu \mathrm{M})$ plus L-cysteine $(200 \mu \mathrm{M})$. All reactions were incubated for $30 \mathrm{~min}$ at room temperature except for the combination of $\mathrm{Zn}-$ Isc $\mathrm{U}$ with IscS and L-cysteine, which was reacted for $5 \mathrm{~min}$. All experiments were repeated at least twice, and similar results were obtained. 

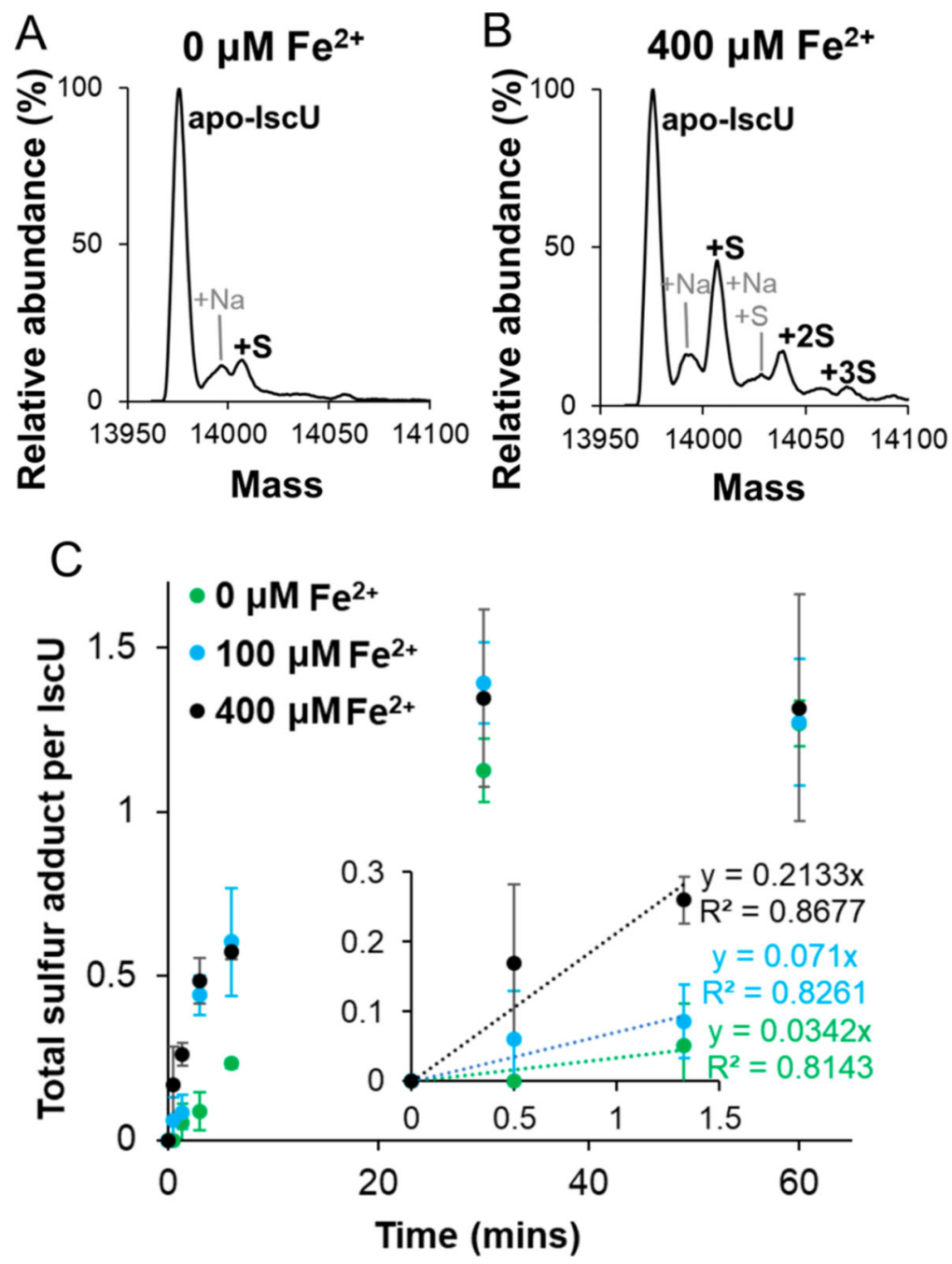

Figure 4.

$\mathrm{Fe}^{2+}$ enhances sulfur transfer from IscS to IscU. Time-resolved acid-quench (1\% formic acid) denaturing MS experiments were used to track sulfur accumulation on IscU after mixing apo-IscU $(75 \mu \mathrm{M})$ with IscS $(15 \mu \mathrm{M})$, L-cysteine $(300 \mu \mathrm{M})$, and various concentrations of ferrous acetate. Deconvoluted zero charge MS spectra are shown for monomeric IscU using (A) $0 \mu \mathrm{M}$ and (B) $400 \mu \mathrm{M}$ ferrous acetate after a reaction time of 3 min. (C) Total accumulated sulfur adducts per IscU are displayed as a function of time. The inset shows the early time points overlaid with linear regression fits (dashed lines). The error bars represent replicate errors $(n=3)$. 

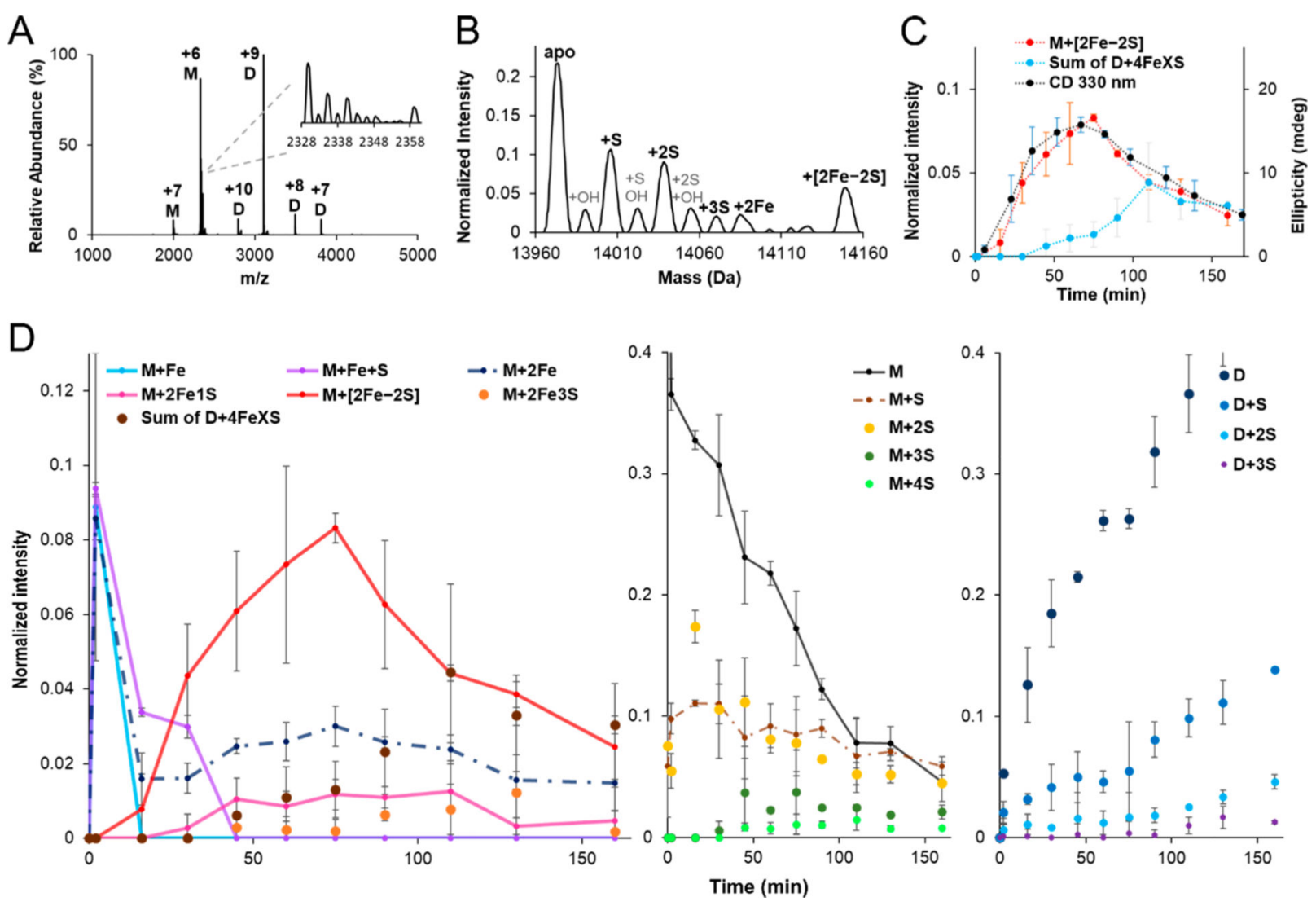

Figure 5.

Time-dependent tracking of intermediates in Fe-S cluster formation by native MS. (A) Representative raw and (B) deconvoluted zero charge MS spectra collected during cluster formation reactions. The inset of (A) shows the raw MS spectrum for the +6 charge state of monomeric IscU (M: monomeric IscU, D: dimeric IscU). Experimental conditions: $100 \mu \mathrm{M}$ apo-IscU, $3 \mu \mathrm{M}$ IscS, $200 \mu \mathrm{M}$ L-cysteine, $100 \mu \mathrm{M}$ GSH, and $250 \mu \mathrm{M}$ ferrous acetate. (C) Comparison of the kinetics of [2Fe-2S]-IscU formation from native MS (normalized intensity; orange) and the CD spectroscopic signature at $330 \mathrm{~nm}$ (ellipticity; blue). The decay of the native MS [2Fe-2S] cluster signal corresponded to the development of four iron species (gray). The error bars are replicate errors $(n=2)$. (D) Normalized intensity of iron (left panel), non-iron monomeric IscU (middle panel), and non-iron dimeric IscU (right panel) related species displayed as a function of time. Proposed intermediates on the main pathway (see Figure 6) are shown with connecting solid lines. Additional proposed intermediates for viable secondary pathways are displayed with connecting dotted lines, whereas proposed off pathway species are shown without lines. 


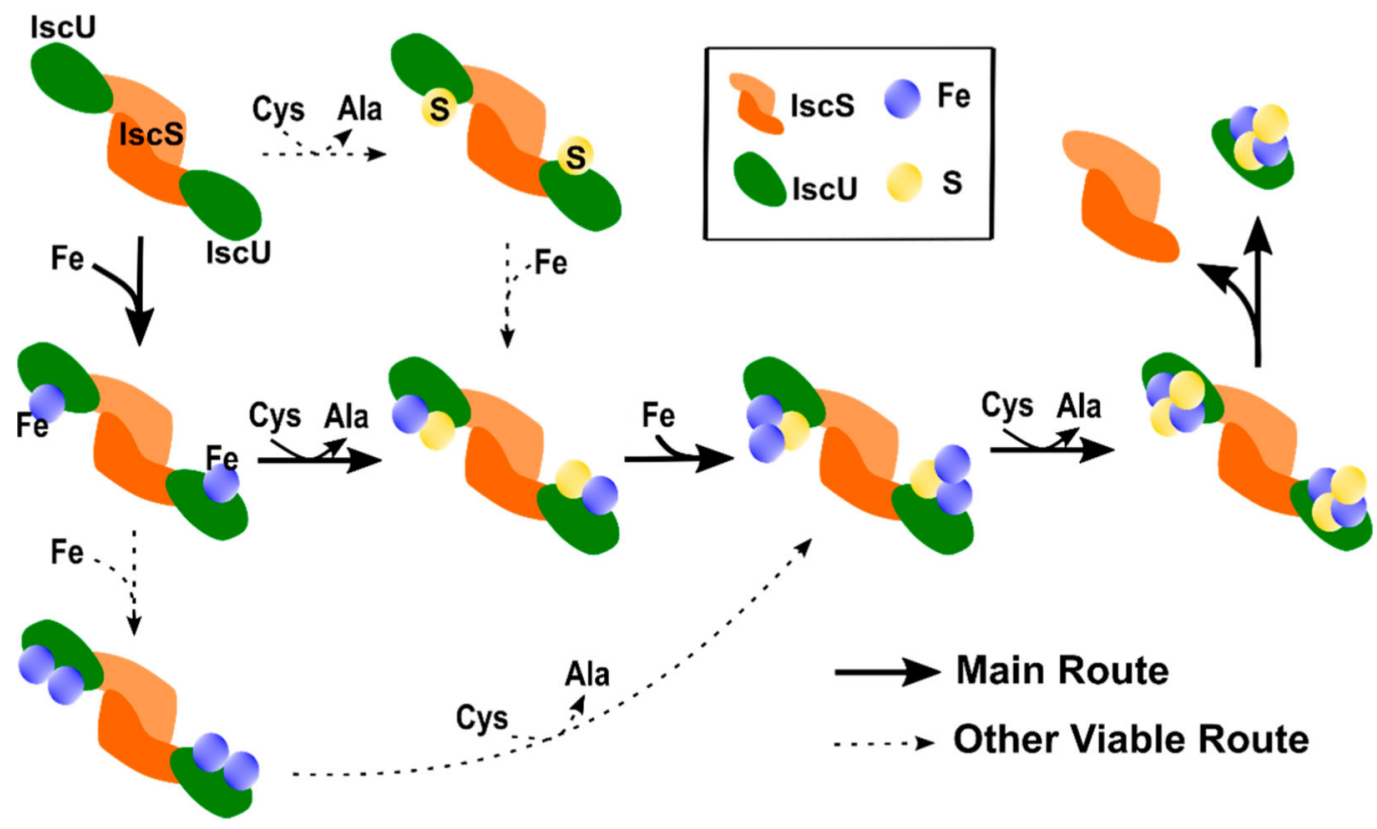

Figure 6.

Proposed mechanism for iron-sulfur cluster assembly on IscU. The primary route is highlighted in bold with additional secondary pathways shown as dashed lines. The proposed mechanism is based on the assumption that the resulting iron and sulfur bound species of IscU are a readout of intermediates on the IscU-IscS complex. 